\title{
Quantum Dissipative Systems and Feedback Control Design by Interconnection
}

\author{
Matthew R. James, Fellow, IEEE, and John E. Gough
}

\begin{abstract}
The purpose of this paper is to extend J.C. Willems' theory of dissipative systems to open quantum systems described by quantum noise models. This theory, which combines ideas from quantum physics and control theory, provides useful methods for analysis and design of dissipative quantum systems. We describe the interaction of the plant and a class of external systems, called exosystems, in terms of feedback networks of interconnected open quantum systems. Our results include an infinitesimal characterization of the dissipation property, which generalizes the well-known Positive Real and Bounded Real Lemmas, and is used to study some properties of quantum dissipative systems. We also show how to formulate control design problems using network models for open quantum systems, which implements Willems" "control by interconnection" for open quantum systems. This control design formulation includes, for example, standard problems of stabilization, regulation, and robust control.
\end{abstract}

Index Terms-Control by interconnection, damping, dissipation, quantum feedback control, quantum feedback networks, quantum noise, regulation, robustness, stabilization.

\section{INTRODUCTION}

$\mathbf{I}$ N 1972 J.C. Willems [37] developed a general theory of dissipative systems for the purpose of stability analysis of open systems, that is, systems that may be subject to external influences, [39]. This theory generalizes Lyapunov methods that apply to closed systems, as well as important results in control theory including the positive and bounded real lemmas. The theory is widely used in control system analysis and design. In particular, methods for stability analysis, e.g., [17], [18], [25], control design by energy shaping, interconnection, robust control system design; e.g. [29], [31], [35], [38]-[40] have been developed. While Willems' theory applies to quite general nonlinear systems, it has its origins in classical physical systems and is based on describing energy storage and flows. As a simple example, consider a passive series RLC circuit. Energy $V=$ $(L / 2) i^{2}+(1 / 2 C) q^{2}$ is stored in the inductor and the capacitor (here, $L$ is the inductance, $C$ is the capacitance, $q$ is capacitor charge, and $i$ is the current). The external voltage $v$ is related to the internal voltages by $v=v_{L}+v_{R}+v_{C}$, with $v_{L}=L \ddot{q}$,

Manuscript received August 27, 2007; revised November 30, 2008, August 19, 2009, and October 27, 2009; accepted March 04, 2010. First published March 22, 2010; current version published July 30, 2010. This work was supported by the Australian Research Council. Recommended by Associate Editor M. Fujita.

M. R. James is with the School of Engineering, Australian National University, Canberra, ACT 0200, Australia (e-mail: matthew.james@anu.edu.au).

J. E. Gough is with the Institute for Mathematical and Physical Sciences, University of Wales, Aberystwyth, Ceredigion, SY23 3BZ, Wales (e-mail: jug@aber.ac.uk).

Digital Object Identifier 10.1109/TAC.2010.2046067
$v_{R}=R \dot{q}$ and $v_{C}=(1 / C) q$, and this determines the dynamical equation $(d i / d t)=(1 / L)(v-R i-(1 / C) q)$. The rate of change of stored energy is given by

$$
\dot{V}=-R i^{2}+v i .
$$

The first term on the right hand side is the energy dissipated by the resistor, and the second term is the power applied to the circuit by an external source. This expression describes the balance of energy flows, and implies the inequality

$$
\dot{V} \leq v i
$$

which is an example (in differential form) of the dissipation inequalities considered by Willems. It says simply that the rate at which energy is stored must be less than the rate at which energy is supplied; the remainder being dissipated. Inequalities of this type are of fundamental importance to stability analysis of open systems. Furthermore, such inequalities can be exploited to facilitate control system design.

In the physics literature, methods have been developed to model energy loss and decoherence (loss of quantum coherence) arising from the interaction of a system with an environment; see, e.g. [6], [8], [21]. These models may be expressed using tools which include completely positive maps, Lindblad generators, and master equations. In the 1980s it became apparent that a wide range open quantum systems, such as those found in quantum optics, could be described within a new unitary framework of quantum stochastic differential equations, [12], [13], [19], where quantum noise is used to represent the influence of large heat baths and boson fields (which includes optical and phonon fields). Completely positive maps, Lindblad generators, and master equations are obtained by taking expectations.

Quantum noise models cover a wide range of situations involving light and matter. In this paper, we use quantum noise models for boson fields, as occur in quantum optics, mesoscopic superconducting circuits, and nanomechanical systems, although many of the ideas could be extended to other contexts. Quantum noise models can be used to describe an optical cavity, which consists of a pair of mirrors (one of which is partially transmitting) supporting a trapped mode of light. This cavity mode may interact with a free external optical field through the partially transmitting mirror. The external field consists of two components: the input field, which is the field before it has interacted with the cavity mode, and the output field, being the field after interaction. The output field may carry away energy, and in this way the cavity system dissipates energy. This quantum system is in some ways analogous to the RLC circuit discussed above, which stores electromagnetic energy in the inductor and capacitor, but loses energy as heat through 
the resistor. The cavity also stores electromagnetic energy, quantized as photons, and these may be lost to the external field. If $V$ denotes the physical observable for the energy of the cavity mode, and if a laser beam is applied as an input $w$, then the energy dissipation relation for the cavity is of the form

$$
\mathcal{G}(V) \leq z^{*} w+w^{*} z
$$

where $z$ is a physical quantity of the cavity (notation is explained at the end of this section). The term $\mathcal{G}(V)$ plays the role of $\dot{V}$, and is discussed further in Sections II-A and II-D (it is the Lindblad generator applied to $V$ ). This inequality relates the rate at which energy is stored in the cavity to the rate at which energy is supplied, with the remainder being lost to the external field (which serves as a heat bath).

Motivated by the need for analysis and design methods for feedback control systems (e.g. [2], [4], [10], [20], [22], [23], [42], [43]) in emerging quantum technologies, the principal goal of this paper is to formalize a notion of dissipation for open quantum systems in a way that is helpful for quantum control analysis and design. This is achieved by supplementing the quantum noise framework discussed above with explicit mechanisms for describing how other quantum systems, called exosystems, may influence the behavior of the system of interest, called the plant. An exosystem may simply represent another system with which the plant interacts, or it may represent unmodeled plant dynamics or other sources of uncertainty. Exosystems provide an explicit physical representation for the disturbance signals used in [20], or as a signal source as in a modulated laser. Controllers (other classical or quantum systems used to control the plant) may also be regarded as exosystems.

Quantum noise models have a natural input-output structure, and so it is natural to interconnect systems by connecting the output field of one system to the input field of another; the simplest case being a cascade or series connection. Based on this type of field-mediated interconnection, a theory of quantum feedback networks (QFN) has been developed [7], [11], [14], [15], [45]-[47]. We use this QFN framework, together with the direct couplings between systems not using fields, to describe how exosystems interact with the plant. The quantum information carried by the fields between systems may be regarded as a "quantum signal", and so the QFN theory provides a framework for coherent control, [23], [28], [45], [46].

The fundamental dissipation inequality we present is expressed in terms of a storage function, and a dissipation inequality (generalizing (2) along the lines of (3)). The storage function is a quantum observable which may simply be a physical quantity related to energy, or some other quantity related to system performance. The dissipation inequality is meant to hold for all exosystems in a specified class (which describes the nature of the external influences being modeled). We provide an infinitesimal characterization of the dissipation property, and this important tool is used indispensably in this paper. This characterization includes (time-domain) generalizations of the well-known Positive Real and Bounded Real Lemmas, and is used to study some properties of quantum dissipative systems. We show that essentially all open quantum systems are dissipative for a suitable choice of supply rate. This "natural" supply rate includes terms corresponding to the classical notion of passivity, a dissipation or damping term due to the quantum noise, and a dissipation term due to the exosystem.

The network description used in this paper turns out to be very appropriate and efficient, and was inspired by the behavioral "control as interconnection" perspective in Willems' more recent work, [38], [39] (also [33], [34], [41]). Indeed, the framework we develop can also be used to describe how systems are influenced by controllers, and hence is useful for control design by interconnection, [38], [39]; see also [29]-[31], [35]. We give a description of how to formulate control design problems in these terms, such as standard problems of stabilization, regulation, and robust control. It is important to appreciate that because we express control design problems in terms of quantum noise models, the controllers obtained can (in principle) be physically realized - this is vital when the controller is to be itself a quantum system, as in coherent control, [20], [22], [23], [27], [28], [43].

Note that issues of stability are important in the analysis and design of quantum networks. This is because quantum networks may contain active elements that introduce energy, and whenever this happens, stability of feedback loops in the network is a basic consideration (small gain theorem, [9], [48], [49]). Energy may be introduced by design, such as via an amplifier, or by accident due to undesirable environmental influences. It also plays a role in regulation of a system to a desired equilibrium mode of operation.

We begin in Section II by describing the mathematical models for quantum feedback networks we use, which are expressed in terms of the quantum stochastic calculus, [12], [19]. This section includes some material aimed at helping orient the reader to the ideas, models and notation used in the remainder of this paper. Our main definitions and results for quantum dissipative systems are given in Section III, which includes some examples for illustration. Section IV contains a formulation of control by interconnection methodology for quantum dissipative systems, which we illustrate using simple examples. The Appendix contains some definitions and results needed in the paper.

Background references. A number of articles and books are available to help readers with the background material on which the present paper is based. The papers [36] and [45] provide excellent introductions to aspects of the quantum models we use. The paper [5] is a tutorial article written to assist control theorists and engineers by providing introductory discussions of quantum mechanics, open quantum stochastic models, and quantum filtering. The book [13] is an invaluable resource for quantum noise models and quantum optics, while the book [32] provides a detailed mathematical treatment of the HudsonParthasarathy theory of the quantum stochastic calculus. The recently published book [44] provides significant physical insight into quantum measurement and feedback. The textbooks [3] and [24] are excellent references on quantum mechanics. The papers [14] and [15] contain the basic results concerning quantum feedback networks used in this paper.

Notation. In this paper we use matrices $M=\left\{m_{i j}\right\}$ with entries $m_{i j}$ that are operators on an underlying Hilbert space. The asterisk $*$ is used to indicate the Hilbert space adjoint $A^{*}$ of an operator $A$, as well as the complex conjugate $z^{*}=x-i y$ of a 
complex number $z=x+i y$ (here, $i=\sqrt{-1}$ and $x, y$ are real). Real and imaginary parts are denoted $\operatorname{Re}(z)=\left(z+z^{*}\right) / 2$ and $\operatorname{Im}(z)=-i\left(z-z^{*}\right) / 2$ respectively. The conjugate transpose $M^{\dagger}$ of a matrix $M$ is defined by $M^{\dagger}=\left\{m_{j i}^{*}\right\}$. Also defined are the conjugate $M^{\#}=\left\{m_{i j}^{*}\right\}$ and transpose $M^{T}=\left\{m_{j i}\right\}$ matrices, so that $M^{\dagger}=\left(M^{T}\right)^{\#}=\left(M^{\#}\right)^{T}$. In the physics literature, it is common to use the dagger $\dagger$ to indicate the Hilbert space adjoint. The commutator of two operators $A, B$ is defined by $[A, B]=A B-B A . \delta(\cdot)$ is the Dirac delta function, and $\delta_{j k}$ is the Kronecker delta. The tensor product of operators $A, B$ defined on Hilbert spaces $\mathrm{H}, \mathrm{G}$ is an operator $A \otimes B$ defined on the Hilbert space $\mathrm{H} \otimes \mathrm{G}$ (tensor product of Hilbert spaces) defined by $(A \otimes B)(\psi \otimes \phi)=(A \psi) \otimes(B \phi)$ for $\psi \in \mathrm{H}$, $\phi \in \mathrm{G}$; we usually follow the standard shorthand and write simply $A B=A \otimes B$ for the tensor product, and also $A=A \otimes I$ and $B=I \otimes B$.

\section{PRELIMINARIES}

\section{A. The Classical RLC Circuit Revisited}

Before embarking on a description of the class of open quantum systems considered in this paper, we revisit the simple classical RLC circuit mentioned in Section I in order to provide some conceptual and notational connections with the quantum theory. We may choose the charge and current as the phase space variables for the circuit, $x=(q, i)^{T}$, which evolve according to the system of equations $(d q / d t)=i$, $(d i / d t)=(1 / L)(v-R i-(1 / C) q)$.

For an arbitrary smooth function $f(x)=f(q, i)$ of the state, we have by the chain rule

$$
\begin{aligned}
\frac{d}{d t} f(x(t))= & i(t) \frac{\partial f}{\partial q}(x(t)) \\
& +\frac{1}{L}\left(v(t)-R i(t)-\frac{1}{C} q(t)\right) \frac{\partial f}{\partial i}(x(t))
\end{aligned}
$$

or in compact form $\dot{f}=\mathcal{G}_{v}(f)$, where $\mathcal{G}_{v}(f)$ is the directional derivative operator $\mathcal{G}_{v}(f)=\nabla f \cdot(i,(1 / L)(v-R i-(1 / C) q))^{T}$. In particular, the stored energy $V=(L / 2) i^{2}+(1 / 2 C) q^{2}$ is a quadratic function of the state variables, and the energy balance (1) is obtained from setting $f=V$

$$
\dot{V}=\mathcal{G}_{v}(V) \equiv r^{0}(v)
$$

where $r^{0}(v)$ is the function $-R i^{2}+i v$ on the phase plane, also dependent on the input voltage $v$.

The energy balance relation (1) and dissipation inequality (2) become

$$
\mathcal{G}_{v}(V)-r^{0}(v)=0
$$

and

$$
\mathcal{G}_{v}(V)-r(v) \leq 0
$$

where the respective supply rates are $r^{0}(v)=-R i^{2}+i v$ and $r(v)=i v$. If we regard the voltage $v$ as an input and the current $i$ as an output function of the phase space variables, then it can be seen that the supply rates are functions of the phase space variables and the input.
Functions $f=f(q, i)$ of the phase space variables correspond to the physical variables of interest, and are basic to any description of classical mechanics. When considering open physical systems, such as the RLC circuit, or the open quantum systems discussed in this paper, it is helpful to have a notation for indicating to which physical system a physical variable belongs. If we write $\mathcal{A}_{R L C}$ for RLC circuit physical variables (the set of smooth functions on the phase space manifold) then this is in fact a commutative algebra which may be extended to a $*$-algebra by taking complex-valued functions with the choice of complex conjugation as the $*$-operation. Likewise we could write $\mathcal{A}_{\text {ex }}$ for functions of the external variables $v$. We then see that the energy function $V$ belongs to $\mathcal{A}_{R L C}$. The supply rates however belong to the algebra of functions over both state variables and external variables, the tensor product $\mathcal{A}_{R L C} \otimes \mathcal{A}_{\text {ex }}$, so that $r^{0}, r \in \mathcal{A}_{R L C} \otimes \mathcal{A}_{\text {ex }}$. In the quantum setting, these algebras will be non-commutative (in fact algebras of operators over Hilbert spaces).

\section{B. Quantum Mechanics}

In quantum mechanics [24] physical quantities like energy, spin, position, etc., are expressed as observables; these are represented as self-adjoint operators acting on a Hilbert space $\mathrm{H}$. Other physical variables, like annihilation operators (see Appendix B)—which are not self-adjoint - are also of importance. We will use the notation $\mathcal{A}$ to refer to the collection of physical variables for a system (in general $\mathcal{A}$ is a non-commutative *-algebra). We refer to $\mathcal{A}$ as the physical variable space for the system. Unit vectors $\psi \in \mathrm{H}$ are called state vectors. When a quantum system is in a state defined by a state vector $\psi \in \mathrm{H}$, the expected value of an observable $A \in \mathcal{A}$ is defined in terms of the Hilbert space inner product: $\langle\psi, A \psi\rangle$. In what follows we use the shorthand notation $\langle A\rangle$ to denote expectation when the underlying state is understood.

The postulates of quantum mechanics state that for a closed system the evolution of states and observables are given in terms of a unitary operator $U(t)$ satisfying the Schrödinger equation

$$
\dot{U}=-i H U
$$

with initial condition $U(0)=I$ (the identity). Here, $H$ is an observable called the Hamiltonian, and represents the energy of the system. State vectors evolve according to $\psi_{t}=U(t) \psi$. Alternatively, we may view state vectors as fixed in time, while observables are taken to evolve according to $A(t)=U^{*}(t) A U(t)$ : this is the Heisenberg picture. Both pictures are equivalent and the average of an observable $A$ in state $\psi$ at time $t$ is given equally by $\langle\psi, A \psi\rangle_{t}=\left\langle\psi_{t}, A \psi_{t}\right\rangle=\langle\psi, A(t) \psi\rangle$.

In this paper we are interested in open quantum systems-systems that interact with other systems or an environment. These systems will be defined in Section II-D in terms of a stochastic generalization of the Schrodinger (8) involving quantum noise. Before considering these open system models, we look at a simple situation of two interacting systems in the next section.

\section{A Pair of Interacting Systems}

Consider a pair of independent systems $P$ (the plant) and $W$ (the exosystem, or signal generator). The physical variable 
spaces for these systems are denoted $\mathcal{A}_{P}$ and $\mathcal{A}_{W}$ respectively, and consist of operators defined on underlying Hilbert spaces $\mathrm{H}_{P}$ and $\mathrm{H}_{W}$ respectively. The physical variable space for the combined system is the tensor product $\mathcal{A}_{P} \otimes \mathcal{A}_{W}$, consisting of operators on the Hilbert space $\mathrm{H}_{P} \otimes \mathrm{H}_{W}$. All operators in $\mathcal{A}_{P}$ may be regarded as operators in $\mathcal{A}_{P} \otimes \mathcal{A}_{W}$ by identifying $A_{P}$ with $A_{P} \otimes I_{W}$, and similarly for $\mathcal{A}_{W}$. As a consequence, all variables in $\mathcal{A}_{P}$ commute with all variables in $\mathcal{A}_{W}$.

Let $H_{P} \in \mathcal{A}_{P}$ and $H_{W} \in \mathcal{A}_{W}$ be the Hamiltonians for each of the systems, respectively; this would be enough to specify their dynamics as isolated, closed, systems. However, we allow them to interact by exchanging energy as specified by an interaction Hamiltonian of the form

$$
H_{P W}=i\left(v^{*} K-K^{*} v\right)
$$

where $K \in \mathcal{A}_{P}$ and $v \in \mathcal{A}_{W}$. The total Hamiltonian for the combined system is $H=H_{P}+H_{P W}+H_{W}$, and the dynamics are given by the Schrödinger (8) using this total Hamiltonian.

Now let's consider the effect of the exosystem $W$ on the plant $P$. Let $V \in \mathcal{A}_{P}$ be a non-negative observable that commutes with $H_{P}$. Then from (8) we see that $V$ evolves according to

$$
\dot{V}=-i[V, H]=v^{*}[V, K]-\left[V, K^{*}\right] v=Z^{*} v+v^{*} Z
$$

where $Z=[V, K] \in \mathcal{A}_{P}$. From this we see that $P$ is lossless, with the right hand side of this relation giving the net rate at which energy is delivered to $P$ from $W$ (cf. [35, eq. (2.39) and Chapter 4], and Section III-A below).

Remark 2.1: While a detailed discussion of physical modeling is beyond the scope of this paper, we briefly discuss a classical analogy to help explain the common form (9) of the interaction Hamiltonian $H_{P W}$ used here. Consider a series LC circuit $P$ connected to an external circuit $W$ (with Hamiltonian $H_{W}$ ), for which the total Hamiltonian is $H=V-q v+H_{W}$, where $V=(L / 2) i^{2}+(1 / 2 C) q^{2}$. In classical mechanics, $V$ evolves according to the equation $\dot{V}=\{V, H\}=\{V,-q\} v=$ $i v{ }^{1}$ The term $-q v$ is the interaction Hamiltonian, with $K=-q$. The quantity $Z$ here is given by $Z=\{V, K\}=i$. Thus with input voltage $v$ and coupling variable $K=-q$, the quantity $Z$ is a conjugate variable, the current $i$. The interaction term $-q v$ may arise as a simplification or approximation of a more detailed description of the interaction.

In the general framework we present in this paper (Section III), $P$ is an open system, and $W$ may be connected to $P$ via field connections in addition to direct couplings of the form (9). Open quantum systems are summarized in Section II-D, and mechanisms for interconnecting them are reviewed in Section II-E.

\section{Definitions}

We consider an open quantum system $G$ with physical variable space $\mathcal{A}_{G}$ consisting of operators $X$ defined on an underlying Hilbert space $\mathrm{H}_{G}$. The self-energy of this system is described by a Hamiltonian $H \in \mathcal{A}_{G}$. This system is driven by a

\footnotetext{
${ }^{1}$ Here, the Poisson bracket is defined by $\{f, g\}=\nabla^{T} f \Sigma \nabla g$, where $\Sigma=$ $\left(\begin{array}{cc}0 & 1 \\ -1 & 0\end{array}\right)$.
}

collection of $n$ field channels given by the quantum stochastic processes

$$
A=\left(\begin{array}{c}
A_{1} \\
\vdots \\
A_{n}
\end{array}\right), \quad \Lambda=\left(\begin{array}{ccc}
A_{11} & \ldots & A_{1 n} \\
\vdots & \vdots & \vdots \\
A_{n 1} & \ldots & A_{n n}
\end{array}\right) .
$$

These respectively describe annihilation of photons in the field channels, and scattering between channels, and are operators on a Hilbert space $F$, with associated variable space $\mathcal{F}$. Specifically, $\mathrm{F}$ is the Hilbert space describing an indefinite number of quanta (called a Fock space [32]), and $\mathcal{F}$ is the space of operators over this space. We assume that these processes are canonical, meaning that we have the following non-vanishing second order Ito products: $d A_{j}(t) d A_{k}(t)^{*}=\delta_{j k} d t$, $d A_{j k}(t) d A_{l}(t)^{*}=\delta_{k l} d A_{j}(t)^{*}, d A_{j}(t) d A_{k l}(t)=\delta_{j k} d A_{l}(t)$ and $d A_{j k}(t) d A_{l m}(t)=\delta_{k l} d A_{j m}(t)$. The simplest situation corresponds to that of a vacuum state $\phi \in \mathrm{F}$ for the field channels, in which case the input processes are purely quantum noise.

Coupling of the system to the field is defined using

$$
S=\left(\begin{array}{ccc}
S_{11} & \ldots & S_{1 n} \\
\vdots & \vdots & \vdots \\
S_{n 1} & \ldots & S_{n n}
\end{array}\right), \quad L=\left(\begin{array}{c}
L_{1} \\
\vdots \\
L_{n}
\end{array}\right)
$$

respectively a scattering matrix with operator entries $S_{i j} \in \mathcal{A}_{G}$ satisfying $S^{\dagger} S=S S^{\dagger}=I$, and a vector of coupling operators $L_{j} \in \mathcal{A}_{G}$.

We use the notation $G=(S, L, H)$ to indicate an open system specified by the parameters $S, L$ and $H$. The Schrodinger equation is

$$
\begin{aligned}
d U(t)=\left\{\operatorname{tr}\left[(S-I) d \Lambda^{T}\right]+\right. & d A^{\dagger} L-L^{\dagger} S d A \\
& \left.-\frac{1}{2} L^{\dagger} L d t-i H d t\right\} U(t)
\end{aligned}
$$

with initial condition $U(0)=I$ determines the unitary motion of the system, in accordance with the fundamental postulate of quantum mechanics. Given a system operator $X \in$ $\mathcal{A}_{G}$, its Heisenberg evolution is defined by $X(t)=\mathrm{j}_{t}(X)=$ $U(t)^{*} X U(t)$ and satisfies

$$
\begin{aligned}
d X(t)= & \left(\mathcal{L}_{L(t)}(X(t))-i[X(t), H(t)]\right) d t \\
& +d A^{\dagger}(t) S^{\dagger}(t)[X(t), L(t)] \\
& +\left[L^{\dagger}(t), X(t)\right] S(t) d A(t) \\
& +\operatorname{tr}\left[\left(S^{\dagger}(t) X(t) S(t)-X(t)\right) d \Lambda^{T}(t)\right] .
\end{aligned}
$$

In this expression, all operators evolve unitarily (e.g. $L(t)=$ $\mathrm{j}_{t}(L)$ ) (commutators of vectors and matrices of operators are defined component-wise), and tr denotes the trace of a matrix. We also employ the notation

$$
\mathcal{L}_{L}(X)=\frac{1}{2} L^{\dagger}[X, L]+\frac{1}{2}\left[L^{\dagger}, X\right] L .
$$

In what follows we write:

$$
\mathcal{G}_{G}(X)=-i[X, H]+\mathcal{L}_{L}(X)
$$

for the generator of the plant $G$. The components of the output fields are defined by $\tilde{A}(t)=\mathrm{j}_{t}(A(t)) \doteq U^{*}(t) A(t) U(t)$, 
$\tilde{\Lambda}(t)=\mathrm{j}_{t}(\Lambda(t)) \doteq U^{*}(t) \Lambda(t) U(t)$ and satisfy the quantum stochastic differential equations

$$
\begin{aligned}
d \tilde{A}(t)= & S(t) d A(t)+L(t) d t \\
d \tilde{\Lambda}(t)= & S^{\#}(t) d \Lambda(t) S^{T}(t)+S^{\#}(t) d A^{\#}(t) L^{T}(t) \\
& +L(t) d A(t) S^{T}(t)+L^{\#}(t) L^{T}(t) d t
\end{aligned}
$$

where $L(t)=\mathrm{j}_{t}(L)$, etc., as above. The output processes also have canonical quantum Ito products.

It can be seen that the parameters $G=(S, L, H)$ provide a compact specification of the open system, assuming canonical field inputs, since they determine the behavior of the system, via the flow $\mathrm{j}_{t}(\cdot)$, as determined by the Schrodinger (10). In the case of a purely static system, we sometimes use the shorthand $S=(S, 0,0)$. Important special cases are $I=(I, 0,0)$, the trivial (identity) system, and $J=(J, 0,0)$, where

$$
J=\left(\begin{array}{ll}
0 & 1 \\
1 & 0
\end{array}\right) .
$$

If $\psi \in \mathrm{H}_{G}$ is an initial system state vector, then with vacuum fields the state vector of the complete system is $\psi \otimes \phi$. Then the quantum expectation $\langle X(t)\rangle$ is defined to be $\langle\psi \otimes \phi, X(t) \psi \otimes \phi\rangle$. In order to describe how quantum noise beyond time $t$ is averaged out, we introduce a collection $\left\{\mathcal{F}_{t}\right\}$ of physical variable spaces such that $\mathcal{F}_{t} \subset \mathcal{A}_{G} \otimes \mathcal{F}$ is generated by operators in $\mathcal{A}_{G}$ and the quantum noises $A_{i j}(s), s \leq t$. Then $X(t)$ is adapted, i.e. $X(t) \in \mathcal{F}_{t}$, and $\mathcal{F}_{0}=\mathcal{A}_{G}$. There is an associated vacuum expectation $\mathbb{E}_{t}: \mathcal{A}_{G} \otimes \mathcal{F} \rightarrow \mathcal{F}_{t}[32$, Chapter 26] with respect to which the open dynamics satisfies

$$
\mathbb{E}_{s}[X(t)]=X(s)+\int_{s}^{t} \mathbb{E}_{s}\left[\mathcal{G}_{G}(X(r))\right] d r
$$

for all $t \geq s$. In this expression $\mathbb{E}_{s}[X(t)]$ depends on the initial operators and the quantum noises up to time $s$, while the noises beyond time $s$ have been averaged out; it captures the Markovian nature of the model.

\section{E. Quantum Feedback Networks}

In this section we describe a quantum framework for feedback networks that will be used in the sequel, [14], [15]. Quantum feedback networks (QFN) consist of open quantum components that are interconnected by means of field channels that serve as "quantum wires". These channels enable the directional transmission of quantum signals, thereby allowing the components to interact; the components may also interact directly via suitable couplings that facilitate bidirectional energy exchanges, as discussed in Section II-C. Here we focus on the directional interconnections. The QFN framework is expressed in terms of elementary constructs that enable efficient description of networks. These network constructs are defined in terms of the open system parameters $(S, L, H)$ discussed in Section II-D. As we will see, the framework generalizes the familiar transfer function descriptions widely used in classical linear systems theory; however, we emphasize that the QFN framework holds for open quantum components whose dynamical variables may

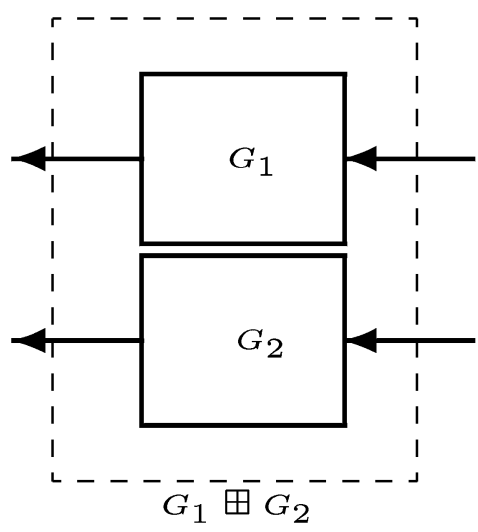

Fig. 1. Concatenation of two systems, $G_{1} \boxplus G_{2}$.

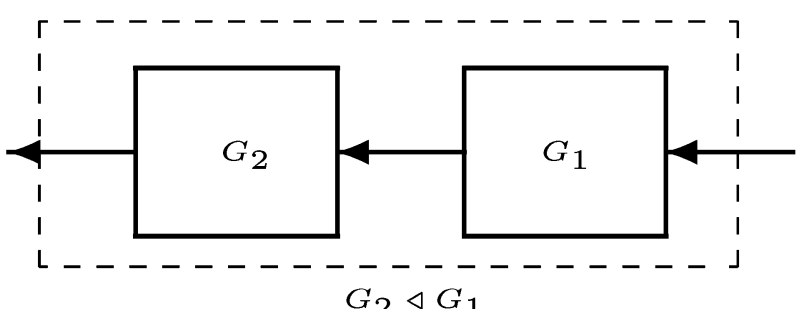

Fig. 2. Series or cascade connection of two systems, $G_{2} \triangleleft G_{1}$.

evolve nonlinearly (by this we mean that the differential equation for a component operator $X(t)$ may be nonlinear).

QFN modeling proceeds as follows. Before implementing any connections, we first collect the components together. This is described using the concatenation product $\boxplus$, Fig. 1. Next, we identify any series connections between components, which we describe using the series product $\triangleleft$, Fig. 2. Networks that can be completely described using the concatenation and series products are called reducible networks (these were studied in detail in [15]). Any remaining signal connections will form part of a feedback loop that can be described in terms of a linear fractional transformation, $F(G)$ [14]. All direct couplings between components can be accommodated using an interaction Hamiltonian of the form (9).

Suppose we are given two such systems: $G_{1}=\left(S_{1}, L_{1}, H_{1}\right)$ and $G_{2}=\left(S_{2}, L_{2}, H_{2}\right)$, with physical variable spaces $\mathcal{A}_{G_{1}}$ and $\mathcal{A}_{G_{2}}$, respectively. The products we define below combine these systems to produce new systems defined in terms of parameters drawn from the tensor product of variable spaces $\mathcal{A}_{G_{1}} \otimes \mathcal{A}_{G_{2}}$.

The concatenation of $G_{1}$ and $G_{2}$ is the system $G_{1} \boxplus G_{2}$ defined by

$$
G_{1} \boxplus G_{2}=\left(\left(\begin{array}{cc}
S_{1} & 0 \\
0 & S_{2}
\end{array}\right),\left(\begin{array}{l}
L_{1} \\
L_{2}
\end{array}\right), H_{1}+H_{2}\right)
$$

as illustrated in Fig. 1 (where each arrowed line may represent multiple channels). It is possible to include zero-dimensional inputs into this scheme as a special case: if a system in isolation has no inputs then it is a closed dynamical system and its dynamics are described by a Hamiltonian $H$. It is convenient just to write this as $G=(-,, H)$ with the absence of inputs denoted by blanks; we then just set $\left({ }_{-}, H_{1}\right) \boxplus\left({ }_{-},{ }_{-}, H_{2}\right):=$ $\left({ }_{-}, H_{1}+H_{2}\right)$ and more generally $\left({ }_{-},-, H_{1}\right) \boxplus\left(S_{2}, L_{2}, H_{2}\right)=$ $\left(S_{2}, L_{2}, H_{2}\right) \boxplus\left({ }_{-}, H_{1}\right):=\left(S_{2}, L_{2}, H_{1}+H_{2}\right)$. 
Now suppose $G_{1}=\left(S_{1}, L_{1}, H_{1}\right)$ and $G_{2}=\left(S_{2}, L_{2}, H_{2}\right)$ have the same number of field channels (i.e. $L_{1}$ and $L_{2}$ have the same length). Then the series product $G_{2} \triangleleft G_{1}$ is defined by

$$
G_{2} \triangleleft G_{1}=\left(S_{2} S_{1}, L_{2}+S_{2} L_{1}, H_{1}+H_{2}+\operatorname{Im}\left\{L_{2}^{\dagger} S_{2} L_{1}\right\}\right)
$$

see Fig. 2.

Several useful facts concerning the series product are the following: (i) given a system $G=(S, L, H)$, we have $G=(I, L, H) \triangleleft(S, 0,0)=(S, 0,0) \triangleleft\left(I, S^{\dagger} L, H\right)$, (ii) the inverse of a system $G$ exists and is given by $G^{-1}=\left(S^{\dagger},-S^{\dagger} L,-H\right)$, by which it is meant that $G^{-1} \triangleleft G=G \triangleleft G^{-1}=I=(I, 0,0)$, and (iii) for any two systems $G_{1}$ and $G_{2}$ we have $G_{2} \triangleleft G_{1}=G_{1} \triangleleft \tilde{G}_{2}$ where $\tilde{G}_{2}=G_{1}^{-1} \triangleleft G_{2} \triangleleft G_{1}=\left(S_{1}^{\dagger} S_{2} S_{1}, S_{1}^{\dagger}\left(S_{2}-I\right) L_{1}+S_{1}^{\dagger} L_{2}, H_{2}+\right.$ $\left.\operatorname{Im}\left\{L_{2}^{\dagger}\left(S_{2}+I\right) L_{1}-L_{1}^{\dagger} S_{2} L_{1}\right\}\right)$.

For future reference, we mention that the generators for the systems formed with the concatenation and series products are

$$
\begin{aligned}
\mathcal{G}_{G_{1} \boxplus G_{2}}(X)= & \mathcal{L}_{L_{1}}(X)+\mathcal{L}_{L_{2}}(X)-i\left[X, q H_{1}+H_{2}\right] \\
= & \mathcal{G}_{G_{1}}(X)+\mathcal{G}_{G_{2}}(X) \\
\mathcal{G}_{G_{2} \triangleleft G_{1}}(X)= & \mathcal{L}_{L_{2}+S_{2} L_{1}}(X) \\
& -i\left[X, H_{1}+H_{2}+\frac{1}{2 i}\left(L_{2}^{\dagger} S_{2} L_{1}-L_{1}^{\dagger} S_{2}^{\dagger} L_{2}\right)\right] \\
= & \mathcal{L}_{S_{2} L_{1}}(X)+\mathcal{L}_{L_{2}}(X)+L_{1}^{\dagger} S_{2}^{\dagger}\left[X, L_{2}\right] \\
& +\left[L_{2}^{\dagger}, X\right] S_{2} L_{1}-i\left[X, H_{1}+H_{2}\right] \\
= & \mathcal{L}_{L_{1}}(X)+\mathcal{L}_{L_{2}}(X)+L_{1}^{\dagger}\left(S_{2}^{\dagger} X S_{2}-X\right) L_{1} \\
& +L_{1}^{\dagger} S_{2}^{\dagger}\left[X, L_{2}\right]+\left[L_{2}^{\dagger}, X\right] S_{2} L_{1} \\
& -i\left[X, H_{1}+H_{2}\right] .
\end{aligned}
$$

Next, consider a system $G$ of the form

$$
G=\left(\left(\begin{array}{ll}
S_{11} & S_{12} \\
S_{21} & S_{22}
\end{array}\right),\left(\begin{array}{c}
L_{1} \\
L_{2}
\end{array}\right), H\right)
$$

The feedback network $F(G)$ defined by Fig. 3 is well-defined provided $I-S_{22}$ is invertible, in which case the parameters for $F(G)$ are given by the linear fractional transformation [14]

$$
\begin{aligned}
F(G)= & \left(S_{11}+S_{12}\left(I-S_{22}\right)^{-1} S_{21},\right. \\
& L_{1}+S_{12}\left(I-S_{22}\right)^{-1} L_{2}, \\
& H+\operatorname{Im}\left\{L_{1}^{\dagger} S_{12}\left(I-S_{22}\right)^{-1} L_{2}\right\} \\
& \left.+\operatorname{Im}\left\{L_{2}^{\dagger} S_{22}\left(I-S_{22}\right)^{-1} L_{2}\right\}\right) .
\end{aligned}
$$

Remark 2.2: Underlying the series and LFT network constructs is the simple idea of equating the input $u_{2}$ with a slightly delayed version of the output $y_{2}$, and then letting the delay tend to zero. Full technical details are given in [13]-[15].

\section{QUANTUM DisSIPATIVE SYSTEMS}

We are now in a position to introduce a definition of dissipation for open quantum systems described by quantum noise models. As we have indicated above, since dissipation concerns the loss of energy or coherence from a system of interest, which we call the plant, to an external environment, or the effect of

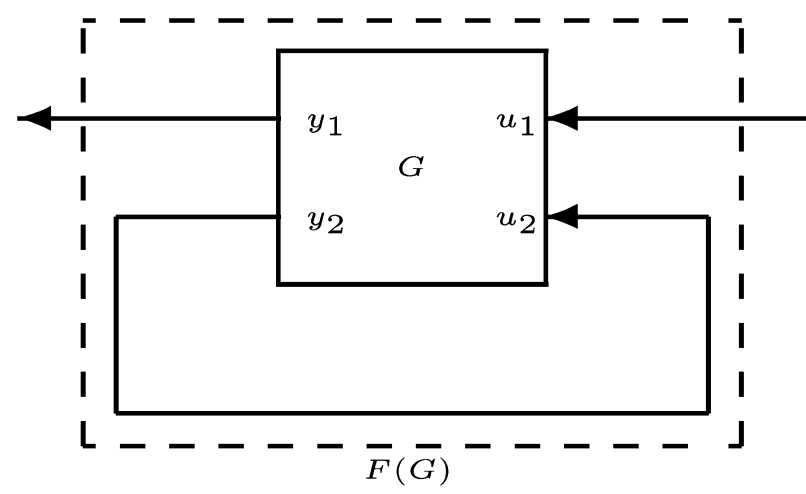

Fig. 3. Quantum feedback network described by the linear fractional transformation $F(G)$.

an external system or environment on the system, we model the external influences as another open system, which we call an $e x$ osystem. Since we wish to consider the effect of a range of exosystems (analogous to a range of signal generators connected to an RLC circuit), we specify a class of allowed exosystems for the dissipation property. The definition, together with some examples, is presented in Section III-A using the QFN framework from Section II-E.

\section{A. Definitions and Differential Characterization}

A plant $P$ is an open system of the type defined in Section II-D

$$
P=(S, L, H) .
$$

It is the system of main interest, and is regarded as being part of a possibly larger network of systems. The space of physical variables for $P$ is denoted $\mathcal{A}_{P}$. Since our interest is in modeling and analyzing the effect of the external influences on the plant, we assume that certain of its input and outputs are available for connection to an exosystem $W$, an open system

$$
W=(R, w, D) .
$$

It is also assumed that certain plant variables are available for direct interconnection with an exosystem. Thus the plant definition and interconnection specification determine how it can interface with exosystems $W$, as discussed in Section II-E. The interconnections determine a network $P \wedge W$, as shown in Fig. 4 (a star product architecture, [14], [16], [49]). In terms of the network constructs of Section II-E, we have explicitly

$$
P \wedge W=F(G) \boxplus H_{P W}
$$

using the LFT (Fig. 3), where

$$
G=(I \boxplus J) \triangleleft(W \boxplus I) \triangleleft(I \boxplus P) \triangleleft(I \boxplus J)
$$

and $H_{P W}$ is a direct interaction Hamiltonian.

We will allow exosystems $W$ to vary in a class $\mathcal{W}$ of such exosystems. The operators constituting the system parameters $W$ belong to an algebra $\mathcal{A}_{P} \otimes \mathcal{A}_{e x}$. The input to the system $P \wedge W$ is assumed to be a canonical vacuum field.

Let $r=r_{P}(W) \in \mathcal{A}_{P} \otimes \mathcal{A}_{e x}$ be a self-adjoint function of the exosystem parameters, depending on the plant parameters (e.g. 


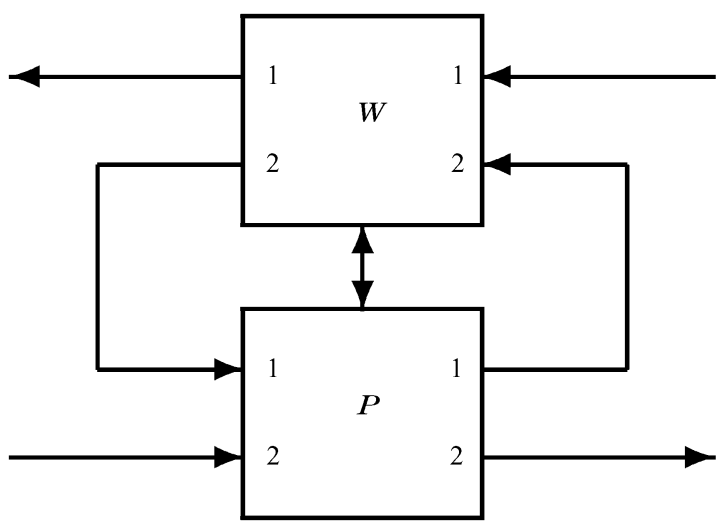

Fig. 4. Plant-exosystem network $P \wedge W$.

a quadratic form), called a supply rate. In general, a supply rate may contain constant terms (see Example 3.10).

We say that the plant $P$ is dissipative with supply rate $r$ with respect to a class $\mathcal{W}$ of exosystems if there exists a non-negative plant observable $V \in \mathcal{A}_{P}$ such that

$$
\mathbb{E}_{0}\left[V(t)-V-\int_{0}^{t} r(W)(s) d s\right] \leq 0
$$

for all exosystems $W \in \mathcal{W}$ and all $t \geq 0$. Here, the dynamics is that of the network $P \wedge W, \mathbb{E}_{0}$ is the initial vacuum expectation onto $\mathcal{A}_{P} \otimes \mathcal{A}_{\text {ex }}$ (which contains $\mathcal{A}_{P}$ ), and $\leq$ denotes operator ordering, see Appendix C.

We shall refer to (25) as the dissipation inequality. It is a natural generalization of Willems' definition [37] to open quantum systems, building on [20]. Following the terminology from [37], when equality holds in (25) for all exosystems $W \in \mathcal{W}$, we say that the plant $P$ is lossless. This terminology refers to the fact that in such cases all energy flows and storages have been accounted for; the total system (plant, exosystem, fields) does not loose energy. Important special cases and applications of the dissipation inequality will be discussed below.

We next present the infinitesimal version of the dissipation inequality (25) in the following theorem.

Theorem 3.1: Let $P$ be a plant, and $P \wedge W$ be a given network structure for a class $\mathcal{W}$ of exosystems. Then the plant $P$ is dissipative with supply rate $r$ with respect to $\mathcal{W}$ if and only if there exists a non-negative plant observable $V \in \mathcal{A}_{P}$ such that

$$
\mathcal{G}_{P \wedge W}(V)-r(W) \leq 0
$$

for all exosystems $W \in \mathcal{W}$.

Proof: Assume $P$ is dissipative as stated, and select any exosystem $W \in \mathcal{W}$ and consider the plant-exosystem network $P \wedge W$. Now apply (16) to the network to obtain

$$
\left.\frac{d}{d t} \mathbb{E}_{0}[V(t)]\right|_{t=0}=\mathcal{G}_{P \wedge W}(V) .
$$

Now combine this with (25) to obtain (26). The converse follows by reversing this argument and integration.

Note that the dissipation inequality (26) involves only plant and exosystem operators, since the LHS depends on operators in the algebra $\mathcal{A}_{P} \otimes \mathcal{A}_{e x}$ (the noise has been averaged out by $\left.\mathbb{E}_{0}\right)$.

Remark 3.2: (Non-Autonomous Case.): For non-autonomous situations, the plant and exosystem operators may depend on time (they can even be random provided they are adapted), and the dissipation inequalities take the forms

$$
\mathbb{E}_{s}\left[V(t)-V(s)-\int_{s}^{t} r(W)(r) d r\right] \leq 0
$$

for all $t \geq s$ and all exosystems $W \in \mathcal{W}$, and

$$
\mathcal{G}_{P \wedge W}(V)(t)-r(W)(t) \leq 0
$$

for all $t \geq 0$ and all exosystems $W \in \mathcal{W}$.

In general we can expect physical systems to be dissipative in some sense, and indeed we characterize this precisely in the next theorem. In what follows we denote by $\mathcal{W}_{u}$ the class of exosystems without any particular constraints (unrestricted) - the only requirement is that members of $\mathcal{W}_{u}$ are open systems that can be connected to the plant. The following simple (but important!) result follows from a calculation similar to that used to prove Theorem 3.1.

Theorem 3.3: Let $P=(S, L, H)$ be a plant, and $P \wedge W$ be a given network structure for the class $\mathcal{W}_{u}$ of (unconstrained) exosystems. Let $V_{0}$ be a non-negative observable, $V_{0} \geq 0$. Then $P$ is lossless (hence dissipative) with respect to $\mathcal{W}_{u}$ for a supply rate $r=r_{0}$ given by

$$
r_{0}(W)=\mathcal{G}_{P \wedge W}\left(V_{0}\right)
$$

with storage function $V=V_{0}$.

Theorems 3.1 and 3.3 contain special cases of interest, e.g. time domain versions of the Bounded and Positive Real Lemmas, as well as fundamental energy balance properties of physical systems. See Theorems 3.6 and 3.7 below.

It is instructive to examine the form of that "natural" supply rate $r_{0}$ (defined by (30)) for the specific interconnection between the plant $P=(I, L, H)$ and exosystems $W=(I, w, D)$ given by the series connection

$$
P \wedge W=P \triangleleft W
$$

and where the Hamiltonian $D$ of the exosystem has the form

$$
D=i\left(v^{\dagger} K-K^{\dagger} v\right)
$$

where $K \in \mathcal{A}_{P}$ is fixed and $v$ commutes with $\mathcal{A}_{P}$ (this provides a mechanism for direct coupling). Let $V_{0}$ be a non-negative observable such that $\left[V_{0}, H\right]=0$. Then we have $r_{0}(W)=$ $r_{0}(w, v)=\mathcal{G}_{P \triangleleft W}\left(V_{0}\right)$, so that the plant $P=(I, L, H)$ satisfies the lossless energy rate relation

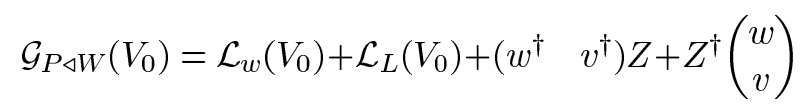

where $Z=\left[V_{0},\left(\begin{array}{l}L \\ K\end{array}\right)\right]$. This relation may be regarded as a generalization of the well-known passivity relation for classical 
Hamiltonian systems (see, e.g. [35, eq. (2.39) and Chapter 4]). If we think of $Z$ as an "output" quantity (not necessarily part of an output field channel!), then the last two terms correspond to a product of input and output terms, i.e. an energy flow into the plant from the exosystem-these terms capture the passivity of the plant, as we now explain. The term $\mathcal{L}_{L}\left(V_{0}\right)$ is known in quantum physics as a "dissipation" or "damping" term, which in the model arises from the quantum noise in the field; when negative, this term represents energy lost from the plant to the field (heat bath). The first term, $\mathcal{L}_{w}\left(V_{0}\right)$, is due to the exosystem $W=(1, w, D)$ and is in general zero when $w$ commutes with plant operators. Indeed, if we integrate (33) and take expectations with respect to a joint state consisting of a plant state vector $\psi_{0}$ for which $\left\langle\psi_{0}, V_{0} \psi_{0}\right\rangle=0$ and the vacuum state for the network input fields, we find that

$$
\begin{aligned}
& \int_{0}^{t}\left\langle\mathcal{L}_{w}\left(V_{0}\right)(s)+\mathcal{L}_{L}\left(V_{0}\right)(s)+\left(w^{\dagger}(s) \quad v^{\dagger}(s)\right) Z(s)+\right. \\
& \left.Z^{\dagger}(s)\left(\begin{array}{c}
w(s) \\
v(s)
\end{array}\right)\right\rangle d s \geq 0
\end{aligned}
$$

which generalizes well known passivity inequalities, [1]. Passivity and gain will be discussed in more detail in Section III-C, which also contains illustrative examples.

\section{B. Stability}

In the case of classical deterministic systems, dissipativity plays an important role in stability analysis, [17], [37]. For instance, strictly passive systems are asymptotically stable. The purpose of this section is to make this connection in our current quantum context using a simple exponential stability criterion, given in the following lemma.

Lemma 3.4: Let $P$ be a plant for which there exists a nonnegative observable $V$ satisfying the differential inequality

$$
\mathcal{G}_{P}(V)+c V \leq \lambda
$$

where $c>0$ and $\lambda$ are real numbers. Then for any plant state we have

$$
\langle V(t)\rangle \leq e^{-c t}\langle V\rangle+\lambda / c
$$

Moreover, if $\lambda=0$ then $\lim _{t \rightarrow \infty}\langle V(t)\rangle=0$.

Proof: Integrating the plant dynamics we find that

$$
\mathbb{E}_{t}\left[V(t+h)-V(t)-c \int_{t}^{t+h} V(r) d r\right] \leq \lambda h, \quad(h \geq 0) .
$$

Now from [32, sec. 26], we have $\mathbb{E}_{0} \mathbb{E}_{t}=\mathbb{E}_{0}$ for $t \geq 0$, hence it follows that the expectation $\langle V(t)\rangle=\left\langle\psi, \mathbb{E}_{0}[V(t)] \psi\right\rangle(\psi$ is the plant state) satisfies

$$
\langle V(t+h)\rangle-\langle V(t)\rangle-c \int_{t}^{t+h}\langle V(r)\rangle d r \leq \lambda h, \quad(h \geq 0) .
$$

From this we deduce $(d / d t)\langle V(t)\rangle \leq-c\langle V(t)\rangle+\lambda$. The assertions of the lemma now follow.

We now state a stability result for strictly dissipative systems.

Theorem 3.5: Let $P$ be dissipative with storage function $V$ and supply rate $r(W)$. Assume that the supply rate satisfies

$$
r(I) \leq-c V
$$

for some real number $c>0$. Then for the unperturbed plant $P=P \triangleleft I$ we have $\lim _{t \rightarrow \infty}\langle V(t)\rangle=0$ for any plant state.

This result follows, of course, from Lemma 3.4 and Theorem 3.1. It is possible to consider more general stability results and this will be taken up in future work.

\section{Passivity and Gain}

In this subsection we consider passivity and gain properties for the series plant-exosystem interconnection, (31). In particular, we present generalizations of the positive and bounded real lemmas (Theorems 3.6 and 3.7 below). To help make the ideas clearer, we will assume that the exosystems commute with the plant. More general results can be formulated using the framework developed in this paper.

Consider a plant $P=(I, L, H)$, and define a class of exosystems $\mathcal{W}_{1}=\left\{W=\left(R, w,-i\left(K^{\dagger} v-v^{\dagger} K\right)\right): K \in\right.$ $\mathcal{A}_{P}$ is fixed and $w, v$ commute with $\left.\mathcal{A}_{P}\right\}$. We say that $P$ is \{lem passive (with respect to $\mathcal{W}_{1}$ ) if it is dissipative with respect to the supply rate

$$
r(W)=-N^{\dagger} N+\left(\begin{array}{ll}
w^{\dagger} & v^{\dagger}
\end{array}\right) Z+Z^{\dagger}\left(\begin{array}{l}
w \\
v
\end{array}\right)+\lambda
$$

for some non-negative real number $\lambda$. Here $N, Z \in \mathcal{A}_{P}$ are plant operators used to specify the supply rate $r(W)$ in (40) which captures the basic passivity relation (recall (1) and (34)). Note that $N$ is related to damping. Explicitly, $P$ is passive if there exists a non-negative system observable $V \in \mathcal{A}_{P}$ and a non-negative real number $\lambda$ such that

$$
\begin{array}{r}
\mathbb{E}_{0}\left[V(t)-V-\int_{0}^{t}\left(\left(\begin{array}{c}
w^{\dagger}(s) \\
v^{\dagger}(s)
\end{array}\right) Z(s)+Z^{\dagger}(s)(w(s) \quad v(s))-\right.\right. \\
\left.\left.N^{\dagger}(s) N(s)\right) d s\right]-\lambda t \leq 0
\end{array}
$$

for all exosystems $W \in \mathcal{W}_{1}$ and all $t \geq 0$.

Theorem 3.6: (Positive Real Lemma): A plant $P=(I, L, H)$ is passive (with respect to $\mathcal{W}_{1}$ ) if and only if there exists a nonnegative system observable $V \in \mathcal{A}_{P}$ and a non-negative real number $\lambda$ such that

$$
\begin{aligned}
\mathcal{L}_{L}(V)-i[V, H]+N^{\dagger} N-\lambda & \leq 0 \\
Z & =\left[V,\left(\begin{array}{l}
L \\
K
\end{array}\right)\right] .
\end{aligned}
$$


Proof: We use some results from the Appendix in what follows. If $P$ is passive, there exists a non-negative system observable $V \in \mathcal{A}_{P}$ and a non-negative real number $\lambda$ such that

$$
\begin{aligned}
& \mathcal{L}_{L}(V)-i[V, H]\left(\begin{array}{c}
w^{\dagger} \\
v^{\dagger}
\end{array}\right)([V, L]-Z)+ \\
& \left(\left[L^{\dagger}, V\right]-Z^{\dagger}\right)\left(\begin{array}{ll}
w & v
\end{array}\right)+N^{\dagger} N-\lambda \leq 0
\end{aligned}
$$

for all exosystem parameters $W=\left(R, w,-i\left(v^{\dagger} K-K^{\dagger} v\right)\right) \in$ $\mathcal{W}_{1}$. Setting $w=0$ and $v=0$ we obtain (42). Now let $w$ vary freely but commuting with plant operators to deduce (43).

The Bounded Real Lemma is used to determine the $L^{2}$ gain of a system, and in conjunction with the Small Gain Theorem, can be used for robust stability analysis and design, [9], [16], [49]. Consider a plant $P=(1, L, H)$, together with the exosystem class $\mathcal{W}_{2}=\left\{W=(R, w, 0): w\right.$ commutes with $\left.\mathcal{A}_{P}\right\}$. We say that $P$ has gain $g$ (with respect to $\mathcal{W}_{2}$ ) if it is dissipative with respect to the supply rate

$$
r(W)=g^{2} w^{\dagger} w-(N+Z w)^{\dagger}(N+Z w)+\lambda
$$

for a real number $\lambda \geq 0$, and class $\mathcal{W}_{2}$; i.e. there exists a nonnegative system observable $V \in \mathcal{A}_{P}$ and a non-negative real number $\lambda$ such that

$$
\mathbb{E}_{0}\left[\begin{array}{r}
t(t)-V-\int_{0}^{t}\left(g^{2} w^{\dagger}(s) w(s)-(N(s)+Z(s) w(s))^{\dagger} \times\right. \\
(N(s)+Z(s) w(s))) d s]-\lambda t \leq 0
\end{array}\right.
$$

for all exosystems $W \in \mathcal{W}_{2}$ and all $t \geq 0$. Here, $g>0$ is a real number (gain parameter), and $N, Z \in \mathcal{A}_{P}$ are plant operators used to specify a performance quantity $N+Z w$ used in the supply rate $r(W)$ in (45).

Theorem 3.7: (Bounded Real Lemma): A plant $P=$ $(I, L, H)$ has gain $g$ (with respect to $\mathcal{W}_{2}$ ) if and only if there exists a non-negative system observable $V \in \mathcal{A}_{P}$ and a non-negative real number $\lambda$ such that

$$
\Gamma=g^{2}-Z^{\dagger} Z \geq 0
$$

and

$$
\begin{aligned}
\mathcal{L}_{L}(V)-i[V, H]-w^{\dagger} \Gamma w+w^{\dagger}\left([V, L]+Z^{\dagger} N\right)+ \\
\left([V, L]+Z^{\dagger} N\right)^{\dagger} w+N^{\dagger} N-\lambda \leq 0
\end{aligned}
$$

for all exosystem parameters $w$. If $\Gamma^{-1}$ exists, then $P=(1, L, H)$ has gain $g$ (with respect to $\mathcal{W}_{2}$ ) if

$$
\begin{aligned}
\mathcal{L}_{L}(V)-i[V, H]+N^{\dagger} N+ \\
\left(\left[L^{\dagger}, V\right]+N^{\dagger} Z\right) \Gamma^{-1}\left([V, L]+Z^{\dagger} N\right)-\lambda \leq 0 .
\end{aligned}
$$

Proof: If $P$ has gain $g$, then there exists a non-negative system observable $V \in \mathcal{A}_{P}$ and a real number $\lambda \geq 0$ such that

$$
\begin{array}{r}
\mathcal{L}_{L}(V)-i[V, H]-w^{\dagger} \Gamma w+w^{\dagger}\left([V, L]+Z^{\dagger} N\right)+ \\
\left([V, L]+Z^{\dagger} N\right)^{\dagger} w+N^{\dagger} N-\lambda \leq 0
\end{array}
$$

for all exosystem parameters $w$. From the Appendix we see that $\Gamma \geq 0$, hence (47). In equality (48) also follows.

Next, if $\Gamma^{-1}$ exists, define $w^{\star}=\Gamma^{-1}\left([V, L]+Z^{\dagger} N\right)$. Then by completion of squares

$$
\begin{aligned}
- & w^{\dagger} \Gamma w+w^{\dagger}\left([V, L]+Z^{\dagger} N\right)+\left(\left[L^{\dagger}, V\right]+N^{\dagger} Z\right) w \\
= & \left(\left[L^{\dagger}, V\right]+N^{\dagger} Z\right) \Gamma^{-1}\left([V, L]+Z^{\dagger} N\right) \\
\leq & -\left(w-w^{\star}\right)^{\dagger} \Gamma\left(w-w^{\star}\right)\left(\left[L^{\dagger}, V\right]+N^{\dagger} Z\right) \\
& \times \Gamma^{-1}\left([V, L]+Z^{\dagger} N\right) .
\end{aligned}
$$

This inequality and (49) imply (48) as required.

Examples 3.9 and 3.10 below illustrate the Positive and Bounded Real Lemmas.

Remark 3.8: Note that the "optimal" exosystem parameter $w^{\star}=\Gamma^{-1}\left([V, L]+Z^{\dagger} N\right)$ belongs to $\mathcal{A}_{P}$, and so does not in general commute with $\mathcal{A}_{P}$, and consequently lies outside the class of exosystems under consideration; i.e., $w^{\star} \notin \mathcal{W}_{2}$ in general.

We conclude this section with several examples. The first example is that of a two-level system, Appendix A.

Example 3.9: (Two-Level Atom): In this example we consider an open two level atom $P=\left(1, \sqrt{\gamma} \sigma_{-},(1 / 2) \omega \sigma_{z}\right)$, where $\sigma_{x}, \sigma_{y}, \sigma_{z}$ denote the Pauli matrices (Appendix A) and $\sigma_{ \pm}=$ $(1 / 2)\left(\sigma_{x} \pm i \sigma_{y}\right)$.

Consider the storage function $V_{0}=\sigma_{1}=(1 / 2)\left(I+\sigma_{z}\right) \geq 0$ (here $I$ is the $2 \times 2$ identity matrix), and $W=(I, w, D)$. Then by (33) we have

$$
\begin{aligned}
r_{0}(W)= & -\gamma \sigma_{1}-\sqrt{\gamma}\left(w^{*} \sigma_{-}+\sigma_{+} w\right)+\mathcal{L}_{w}\left(\sigma_{1}\right)-i\left[\sigma_{1}, D\right] \\
= & -\left(\sqrt{\gamma} \sigma_{-}+w\right)^{*}\left(\sqrt{\gamma} \sigma_{-}+w\right)+w^{*} w+\mathcal{L}_{w}\left(\sigma_{1}\right) \\
& -i\left[\sigma_{1}, D\right] .
\end{aligned}
$$

Therefore this system is lossless (passive, recall (40), with $N=$ $\sqrt{\gamma \sigma_{1}}$ and $Z=-\sqrt{\gamma} \sigma_{1}$ ), and has gain 1 with respect to the output quantity $\sqrt{\gamma} \sigma_{-}+w$ (recall (45), with $N=\sqrt{\gamma} \sigma_{-}$and $Z=1)$, and commuting inputs $w$. When $W=(1,0,0)$, by Theorem 3.5 the expected value of $V_{0}(t)=\sigma_{1}(t)$ tends to zero (asymptotically stable).

The next example looks at open quantum oscillators, Appendix B.

Example 3.10: (Open Oscillator): Consider the plant $P=\left(1, \alpha a+\beta a^{*}, \omega a^{*} a\right)$, where $a$ is the annihilation operator (satisfying the commutation relations $\left[a, a^{*}\right]=1$, recall Appendix B), $\alpha$ and $\beta$ are complex numbers, and $\omega$ is a frequency parameter. The case $\alpha=\sqrt{\gamma}, \beta=0$ corresponds to a damped cavity with coupling $L=\sqrt{\gamma} a$, while the undamped oscillator model for an atom using in [10] has coupling $L=\sqrt{\kappa}\left(a+a^{*}\right)$ for which $\alpha=\beta=\sqrt{\kappa}$. The coupling $L=a^{*}$ arises in amplifier models, [13].

With $V_{0}=a^{*} a$ and $W=(1, w, 0)$, from (33) we have

$$
r_{0}(W)=\mathcal{L}_{w}\left(V_{0}\right)+\left(|\beta|^{2}-|\alpha|^{2}\right) V_{0}+w^{*} Z+Z^{*} w+|\beta|^{2}
$$

where $Z=-\alpha a+\beta a^{*}$. From this it can be seen that $P$ is passive whenever $|\beta|^{2}-|\alpha|^{2} \leq 0$ (in which case $N=\sqrt{\left(|\alpha|^{2}-|\beta|^{2}\right) a^{*} a}$ in (40)). Furthermore, if $|\beta|^{2}-|\alpha|^{2}<0$ (strict passivity) and $W=(1,0,0)$ it follows from Lemma 3.4 that the plant is stable, i.e. the expected value 
of $V_{0}$ remains bounded. If in addition $\beta=0$ then the expected value of $V_{0}$ tends to zero.

In the strictly passive case we may complete the squares to deduce that the plant has finite gain. For instance, if $\alpha=\sqrt{\gamma}$, $\beta=0$ (damped cavity) we have

$$
r_{0}(W)=-(\sqrt{\gamma} a+w)^{*}(\sqrt{\gamma} a+w)+w^{*} w+\mathcal{L}_{w}\left(V_{0}\right)
$$

and hence the system has gain 1 relative to the output quantity $\sqrt{\gamma} a+w$ and commuting inputs $w(Z=\sqrt{\gamma} a$ and $Z=1$ in (45)).

If $|\beta|^{2}-|\alpha|^{2}>0$, the plant is not passive and not stable. The case $|\beta|^{2}-|\alpha|^{2}=0$ is marginally stable.

\section{Dissipative Networks}

Dissipation properties for QFNs can be analyzed by considering how the network is constructed in terms of the elementary constructs (concatenation, series connection, direct interaction, LFT) and knowledge of how dissipation "transforms" under these constructs. This is the purpose of the following results. The first lemma considers concatenation and series connections.

Lemma 3.11: Let $P_{1}$ and $P_{2}$ be given plants and $P_{1} \wedge W_{1}$ and $P_{2} \wedge W_{2}$ are network structures to be specified. Assume $P_{1}$ and $P_{2}$ are dissipative with respect to supply rates $r_{P_{1}}\left(W_{1}\right)$ and $r_{P_{2}}\left(W_{2}\right)$, storage functions $V_{1}$ and $V_{2}$, and exosystem classes $\mathcal{W}_{1}$ and $\mathcal{W}_{2}$ respectively. Then:

1) If $P_{1} \wedge W_{1}=P_{1} \boxplus W_{1}$ and $P_{2} \wedge W_{2}=P_{2} \boxplus W_{2}$, then the network $P_{1} \boxplus P_{2}$ is dissipative with respect to the network structure $\left(P_{1} \boxplus P_{2}\right) \triangleleft W$ and exosystem class $\mathcal{W}_{1} \boxplus \mathcal{W}_{2}$; the storage function is $V=V_{1}+V_{2}$ and the supply rate is

$$
\begin{aligned}
r_{P_{1} \boxplus P_{2}}\left(W_{1} \boxplus W_{2}\right)=r_{P_{1}}\left(W_{1}\right)+r_{P_{2}}( & \left.W_{2}\right)+\mathcal{G}_{P_{1} \triangleleft W_{1}}\left(V_{2}\right) \\
& +\mathcal{G}_{P_{2} \triangleleft W_{2}}\left(V_{1}\right) .
\end{aligned}
$$

If $V_{1}$ commutes with $\mathcal{A}_{P_{2}}$ and $\mathcal{W}_{2}$ and if $V_{2}$ commutes with $\mathcal{A}_{P_{1}}$ and $\mathcal{W}_{1}$, then then the supply rate is simply the sum of the supply rates: $r_{P_{1} \boxplus P_{2}}\left(W_{1} \boxplus W_{2}\right)=r_{P_{1}}\left(W_{1}\right)+$ $r_{P_{2}}\left(W_{2}\right)$.

2) If $P_{1} \wedge W_{1}=P_{1} \triangleleft W_{1}$ and $P_{2} \wedge W_{2}=P_{2} \triangleleft W_{2}$, then the network $P_{2} \triangleleft P_{1}$ is dissipative with respect to the network structure $P_{2} \triangleleft P_{1} \triangleleft W$ and exosystem class

$\mathcal{W}=\left\{W: P_{2}^{\prime} \triangleleft W \in \mathcal{W}_{1} \quad\right.$ and $\left.\quad P_{1} \triangleleft W \in \mathcal{W}_{2}\right\}$

where $P_{2}^{\prime}=P_{1}^{-1} \triangleleft P_{2} \triangleleft P_{1}=\left(S_{1}^{\dagger} S_{2} S_{1}, S_{1}^{\dagger}\left(S_{2}-I\right) L_{1}+\right.$ $\left.S_{1}^{\dagger} L_{2}, H_{2}+\operatorname{Im}\left\{L_{2}^{\dagger}\left(S_{2}+I\right) L_{1}-L_{1}^{\dagger} S_{2} L_{1}\right\}\right)$; the storage function is $V=V_{1}+V_{2}$ and the supply rate is

$$
r_{P_{2} \triangleleft P_{1}}(W)=r_{P_{1}}\left(P_{2}^{\prime} \triangleleft W\right)+r_{P_{2}}\left(P_{1} \triangleleft W\right) .
$$

Proof: For part 1, we have

$$
\begin{aligned}
\left.\mathcal{G}_{(} P_{1} \boxplus P_{2}\right) \triangleleft\left(W_{1} \boxplus W_{2}\right) & \left(V_{1}+V_{2}\right) \\
= & \mathcal{G}_{\left(P_{1} \triangleleft W_{1}\right) \boxplus\left(P_{2} \triangleleft W_{2}\right)}\left(V_{1}+V_{2}\right) \\
= & \mathcal{G}_{P_{1} \triangleleft W_{1}}\left(V_{1}\right)+\mathcal{G}_{P_{2} \triangleleft W_{2}}\left(V_{2}\right)+\mathcal{G}_{P_{1} \triangleleft W_{1}}\left(V_{2}\right) \\
& +\mathcal{G}_{P_{2} \triangleleft W_{2}}\left(V_{1}\right) \\
\leq & r_{P_{1}}\left(W_{1}\right)+r_{P_{2}}\left(W_{2}\right)+\mathcal{G}_{P_{1} \triangleleft W_{1}}\left(V_{2}\right)+\mathcal{G}_{P_{2} \triangleleft W_{2}}\left(V_{1}\right)
\end{aligned}
$$

so that the last line is a supply rate for the concatenated system. Similarly, for part 2 we have

$$
\begin{aligned}
\mathcal{G}_{P_{2} \triangleleft P_{1} \triangleleft W}\left(V_{1}+V_{2}\right) & =\mathcal{G}_{P_{2} \triangleleft P_{1} \triangleleft W}\left(V_{1}\right)+\mathcal{G}_{P_{2} \triangleleft P_{1} \triangleleft W}\left(V_{2}\right) \\
& =\mathcal{G}_{P_{1} \triangleleft\left(P_{2}^{\prime} \triangleleft W\right)}\left(V_{1}\right)+\mathcal{G}_{P_{2} \triangleleft\left(P_{1} \triangleleft W\right)}\left(V_{2}\right) \\
& \leq r_{P_{1}}\left(P_{2}^{\prime} \triangleleft W\right)+r_{P_{2}}\left(P_{1} \triangleleft W\right)
\end{aligned}
$$

where $P_{2}^{\prime}=P_{1}^{-1} \triangleleft P_{2} \triangleleft P_{1}=\left(S_{1}^{\dagger} S_{2} S_{1}, S_{1}^{\dagger}\left(S_{2}-I\right) L_{1}+\right.$ $\left.S_{1}^{\dagger} L_{2}, H_{2}+\operatorname{Im}\left\{L_{2}^{\dagger}\left(S_{2}+I\right) L_{1}-L_{1}^{\dagger} S_{2} L_{1}\right\}\right)$ ([15, Theorem 3.4]).

In the next lemma, we consider the dissipation properties of a LFT feedback system in terms of series plant-exosystem network structures.

Lemma 3.12: Let $P$ be a plant of the form (20) that is dissipative with supply rate $r(W)$ and storage function $V$ with respect to the network structure $P \triangleleft W$ and exosystem class $\mathcal{W}$. Assume the LFT system $F(P)$ is well-defined (Fig. 3, Section II-E). Define a class $F(\mathcal{W})=\{(1, w, 0):(1, w, 0) \boxplus$ $\left(1,\left(I-S_{22}\right)^{-1}\left(S_{21} w+L_{2}\right), 0\right) \in \mathcal{W}$. Then $F(P)$ is dissipative with storage function $V$ and supply rate

$r_{F(P)}(W)=r_{P}\left((1, w, 0) \boxplus\left(1,\left(I-S_{22}\right)^{-1}\left(S_{21} w+L_{2}\right), 0\right)\right)$

for $W=(1, w, 0) \in F(\mathcal{W})$ relative to the network structure $F(P) \triangleleft W$.

Proof: Let $W=(1, w, 0) \in F(\mathcal{W})$, and consider the system $F(P) \triangleleft W$. By elimination of the internal signal in the feedback loop, we see that

$$
\tilde{W}=(1, w, 0) \boxplus\left(1,\left(I-S_{22}\right)^{-1}\left(S_{21} w+L_{2}\right), 0\right)
$$

is an admissible exosystem for $P$. The result now follows from the assumed dissipation property for $P$.

The next lemma describes how series architectures may be used to modify the plant and supply rates.

Lemma 3.13: Let $P$ and $Q$ be systems for which the series connection $P \triangleleft Q$ is well defined, and assume

$W \in \mathcal{W}$ implies $Q \triangleleft W \quad$ and $\quad Q^{-1} \triangleleft W \in \mathcal{W}$.

Then the plant $P$ is dissipative with storage function $V$ and supply rate $r_{P}(W)$ with respect to $\mathcal{W}$ and series network architecture $P \triangleleft W$ if and only if the plant $P \triangleleft Q$ is dissipative with storage function $V$ and supply rate $r_{P}(Q \triangleleft W)$ with respect to $\mathcal{W}$ and series network architecture $P \triangleleft Q \triangleleft W$.

Proof: The assertions follow from the relation $\mathcal{G}_{P \triangleleft Q \triangleleft W}(V)=r_{P}(Q \triangleleft W)$.

As a consequence of Lemma 3.13, the scattering matrix $S$ for the plant can be moved into the supply rate.

Corollary 3.14: Let $\mathcal{W}$ be a class of exosystems satisfying

$W \in \mathcal{W}$ implies $(S, 0,0) \triangleleft W \quad$ and $\quad\left(S^{\dagger}, 0,0\right) \triangleleft W \in \mathcal{W}$.

Then:

1) The plant $P=(S, L, H)$ is dissipative with supply rate $r(W)$ with respect to $\mathcal{W}$ series architecture $P \triangleleft W$ if and only if the plant $P^{\prime}=(1, L, H)$ is dissipative with supply rate $r^{\prime}(W)=r\left(\left(S^{\dagger}, 0,0\right) \triangleleft W\right)$ with respect to $\mathcal{W}$ and series architecture $P^{\prime} \triangleleft W$. 


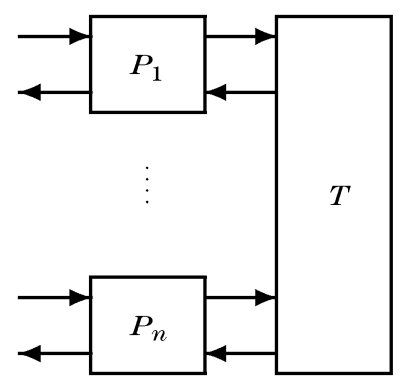

(a)

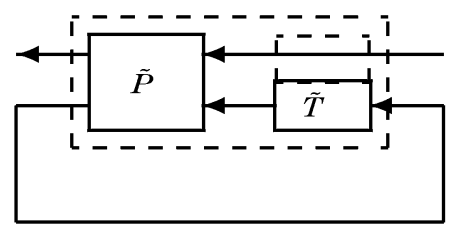

(b)
Fig. 5. (a) Quantum network $N$ formed by interconnecting systems with a static connection system $T$ ([37, Fig. 1]). (b) Equivalent representation of the network $N=F(\tilde{P} \triangleleft(I \boxplus \tilde{T}))$.

2) The plant $P=(I, L, H)$ is dissipative with supply rate $r(W)$ with respect to $\mathcal{W}$ series architecture $P \triangleleft W$ if and only if the plant $P^{\prime}=(S, L, H)$ is dissipative with supply rate $r^{\prime}(W)=r((S, 0,0) \triangleleft W)$ with respect to $\mathcal{W}$ and series architecture $P^{\prime} \triangleleft W$.

The following example illustrates the use of some of the above results by considering the dissipation properties of a network analogous to [37, Fig. 1 and Theorem 5, sec. 4].

Example 3.15: Consider a network $N$ consisting of $n$ plants $P_{1}, \ldots, P_{n}$ interconnected via field channels and a static connection system $T$ (with complex numerical entries), Fig. 5. In terms of the notation of this paper, $N=F(P)$, where $P=$ $\tilde{P} \triangleleft(I \boxplus \tilde{T}), \tilde{P}=Q_{2} \triangleleft\left(P_{1} \boxplus \cdots \boxplus P_{n}\right) \triangleleft Q_{1}$, and $\tilde{T}=Q_{4} \triangleleft T \triangleleft Q_{3}$. Here, $Q_{1}, Q_{2}, Q_{3}, Q_{4}$ are appropriately sized permutation matrices (static components that interchange signals).

We assume that the network $N$ of Fig. 5 is well-defined and that for $j=1, \ldots, n$ let $P_{j}=\left(I, L_{j}, H_{j}\right)$ be dissipative systems with supply rates $r_{j}\left(\tilde{W}_{j}\right)$ and storage functions $V_{j}$ with respect to the network structures $P_{j} \triangleleft \tilde{W}_{j}$ and exosystem classes $\tilde{\mathcal{W}}_{j}$. Since the network $N=F(P)$ has $n$ input channels corresponding to the channels not involved in the interconnection, we may consider a series architecture $N \triangleleft W$, where $W=$ $\boxplus_{j} W_{j}$ with appropriately sized exosystems of the form $W_{j}=$ $\left(1, w_{j}, 0\right)$. Then we can see that with respect to the network structure $N \triangleleft W$ the network $N$ is dissipative with storage function $V=V_{1}+\ldots+V_{n}$ as follows.

First, by the first part of Lemma 3.11, we know that $\boxplus_{j} P_{j}$ is dissipative with storage function $V$ and supply rate $r_{\boxplus_{j} P_{j}}\left(\boxplus \tilde{W}_{j}\right)=\sum_{j} r_{P_{j}}\left(\tilde{W}_{j}\right)$ with respect to the network structure $\left(\boxplus_{j} P_{j}\right) \triangleleft\left(\boxplus_{j} \tilde{W}_{j}\right)$, where $\tilde{W}_{j} \in \tilde{\mathcal{W}}_{j}$. Next, we write $P=R \triangleleft S$ where

$R=Q_{2} \triangleleft\left(\boxplus_{j} P_{j}\right)=\boxplus_{j} R_{j}, \quad S=Q_{1} \triangleleft\left(I \boxplus\left(Q_{4} \triangleleft T \triangleleft Q_{3}\right)\right)$

and $R_{j}=\left(I, L_{\pi_{2}(j)}, 0\right)$. Here $\pi_{2}$ is the permutation corresponding to $Q_{2}$. Now $R$ is simply a re-arrangement of $\boxplus_{j} P_{j}$, and so it is dissipative with supply rate $r_{R}(\tilde{W})=\sum_{j} r_{P_{\pi_{2}(j)}}\left(\tilde{W}_{j}\right)$, where $\tilde{W}=\boxplus \tilde{W}_{j}$, storage function $V$ and series architecture.

Write $\hat{W}=\boxplus_{j} \hat{W}_{j}=\boxplus_{j}\left(I, \hat{w}_{j}, 0\right)$ and $\tilde{W}=S \triangleleft \hat{W}=$ $(1, S \hat{w}, 0)$. Then $P=R \triangleleft S$ is dissipative with respect to the series structure $P \triangleleft \hat{W}$ with supply rate

$$
r_{P}(\hat{W})=r_{R}(\tilde{W})=\sum_{j} r_{P_{\pi_{2}(j)}}\left(\sum_{k} S_{j k} w_{k}\right) .
$$

The supply rate for the network $N=F(P)$ now follows from Lemma 3.12. The exosystem class can be determined from these calculations.

\section{E. Uncertainty Modeling}

Because of its importance to questions of robustness, in this subsection we briefly discuss how uncertainty can be modeled in the framework of this paper. However, a detailed treatment of robustness is beyond the scope of the present paper. The plant-exosystem network architecture $P \wedge W$ illustrated in Fig. 4 together with a specification of exosystem class $\mathcal{W}$ provides a scheme capable of accommodating a wide range of uncertainty models. Here for definiteness we set $P \wedge W=P \triangleleft W$, and consider a simple but common situation of parameter uncertainty.

Consider a plant $P=(I, L, H)$, where $L=(1+\epsilon) L_{0}, H=$ $H_{0}+D$. Here $\epsilon$ is a real parameter. Then using the definition of the series product we can write $P=P_{0} \triangleleft W=\left(I, L_{0}, H\right) \triangleleft$ $\left(I, \epsilon L_{0}, D\right)$, which represents the plant $P$ as a nominal system $P_{0}$ with the uncertainty connected into it from an exosystem $W$ containing the uncertainty terms. Note that $P$ and $W$ are not independent systems, and in fact share variables.

The following example looks at the effect of parameter uncertainty on the behavior of the damped oscillator (recall Example 3.10).

Example 3.16: (Parameter Uncertainty): As an example of this type of uncertainty modeling, consider the plant of Example 3.10 , where $\alpha=\sqrt{\gamma}$, and $\beta=0$ (damped oscillator). Uncertainty arises from the parameter values $\gamma$ and $\omega$, which may not be known accurately. This type of uncertainty is common in quantum optics, where $\gamma$ is a measure of mirror transmissivity and $\omega$ corresponds to a detuning of the cavity relative to the input field. In the above notation, let $L_{0}=\sqrt{\gamma_{0}} a, H_{0}=$ 0 denote the nominal parameters, defining the nominal plant $P_{0}=\left(1, \sqrt{\gamma_{0}} a, 0\right)$, a tuned cavity. Let the true parameters be $L=(1+\epsilon) \sqrt{\gamma_{0}} a, H=\omega a^{*} a$, a detuned cavity $P=$ $\left(1,(1+\epsilon) \sqrt{\gamma_{0}} a, \omega a^{*} a\right)$. Hence the uncertain exosystem is $W=$ $\left(1, \epsilon \sqrt{\gamma_{0}} a, \omega a^{*} a\right.$ ) (so that $w=\epsilon \sqrt{\gamma_{0}} a$ and $D=\omega a^{*} a$ ).

Then a straightforward calculation using (13) shows that

$$
\begin{aligned}
\mathcal{G}_{P}\left(a^{*} a\right) & =\mathcal{G}_{P_{0}}\left(a^{*} a\right)-\left(\epsilon^{2}+2 \epsilon\right) \gamma_{0} a^{*} a \\
& =-(1+\epsilon)^{2} a^{*} a \leq 0 .
\end{aligned}
$$

This means that the true system is always stable, regardless of the uncertainty, though the decay rate does change. This of course is expected of a passive physical system.

However, other aspects of system performance may be affected. For instance, consider the quadratures $q=a+a^{*}$, $p=-i\left(a-a^{*}\right)$, which are rotated by the detuning $\omega$. Indeed, if we focus on the observable $q^{2}$, then the uncertain exosystem contribution to the true generator (recall (13)) is $-i\left[q^{2}, \omega a^{*} a\right]=$ $\omega(q p+p q)$, which depends on the detuning parameter $\omega$. We see, therefore, that a detailed robustness analysis with respect to parameter uncertainty may involve consideration of one or more physical variables. 


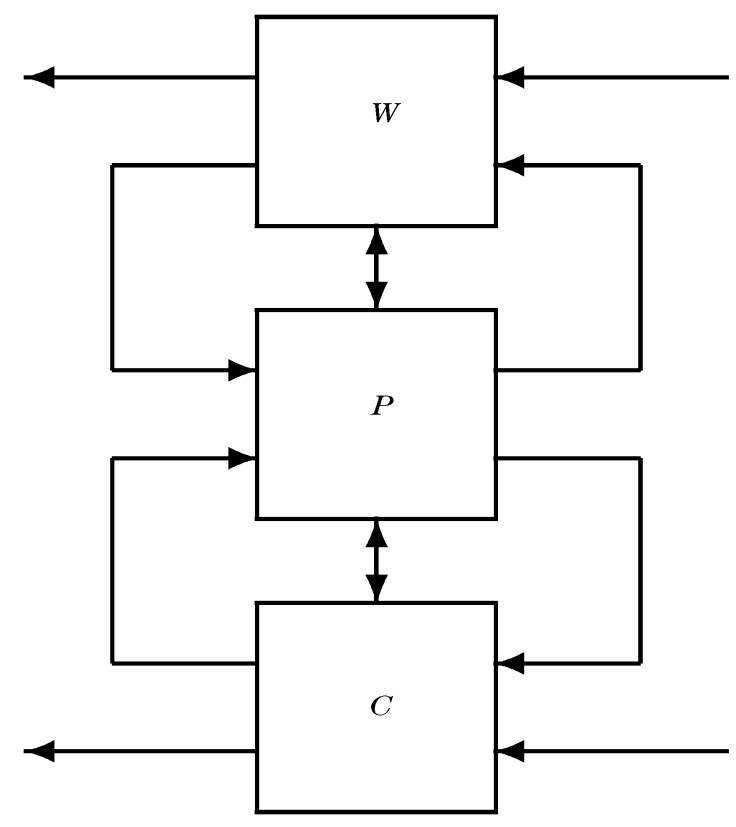

Fig. 6. Plant-controller-exosystem network $P \wedge C \wedge W$.

\section{FeEdBACK Control Design by InterconNeCtion}

\section{A. Control Design Formulation}

In the previous section we described how the behavior of plant is influenced by exogenous influences (represented as exosystems) using a quantum network framework. We now employ this same framework to consider problems of control system design. The network framework allows us to efficiently express a range of control design problems. Indeed, in what follows we describe a control design procedure that extends classical methods including passivity-based control (PBC), "energy shaping" control, interconnection and damping assignment (IDA) PBC, and "control as interconnection", [29]-[31], [33]-[35], [38], [39], [41].

Let $P$ be a plant which can be interconnected with a controller $C$ and an exosystem $W$ in a network $P \wedge C \wedge W$, Fig. 6, as described in Section II-E. Given an exosystem class $\mathcal{W}_{d}$ we denote by $\mathcal{C}$ a class of control systems for which such a network is defined. Since the network parameters $P \wedge C \wedge W$ (together with the inputs) determine the dynamical evolution of the plant-controller-exosystem network, control design can be thought of in terms of shaping the network dynamics in a desired way by appropriate choice of controller $C \in \mathcal{C}$. Note that this framework is general enough to allow us to consider choices for the controller that are quantum, or classical, or a mixture of the two (cf. [20]).

It is worth remarking that the distinction between plant and controller is not always clear-cut. Indeed, the plant may need to be engineered in an appropriate way to facilitate control. For example, one may desire certain field couplings or direct interaction Hamiltonians to be physically available - this clearly relates to the design of the plant. Also, if one is interested in the expected behavior of the network for a range of variables $X$, then it may be appropriate to work directly with the network generator $\mathcal{G}_{P \wedge C \wedge W}(X)$, and use the form of this generator to determine the controller $C$, given the objectives. This approach is roughly dual to a method based on master equations already in use, see, e.g. [33].

We specify the control objectives by encoding them in a nonnegative observable $V_{d} \in \mathcal{A}_{P} \otimes \mathcal{A}_{c}$ (the parameters of the controllers $C \in \mathcal{C}$ are assumed to belong to $\mathcal{A}_{c}$ ), a supply rate $r_{d}(W)$, and a class of exosystems $\mathcal{W}_{d}$ for which a network $(P \wedge C) \wedge W$ is well defined. One then seeks to find, if possible, a controller $C \in \mathcal{C}$ such that

$$
\mathcal{G}_{P \wedge C \wedge W}\left(V_{d}\right)-r_{d}(W) \leq 0
$$

for all exosystems $W \in \mathcal{W}_{d}$. In other words, one seeks a controller for which the closed loop system is dissipative with storage function $V_{d}$, supply rate $r_{d}(W)$, and exosystem class $\mathcal{W}_{d}$. The exosystems are included to facilitate robust control system design for situations where uncertainty and disturbances are important. The observable $V_{d}$ is something which on average should be small (such as regulation errors), or tend to zero as time evolves. The supply rate may also contain such "small" quantities, as well as terms from the exosystems. The supply rate need not be the natural supply rate for the network-the inequality in the dissipation inequality can be exploited to permit other choices.

\section{B. Controller Synthesis}

We shall now describe how standard problems of stabilization, regulation, and robust control fall within the scope of the controller synthesis framework formulated in the previous subsection. We begin with a general synthesis problem that abstracts stabilization and regulation, since they are closely related. These problems correspond to a choice $V_{d}$ of a non-negative observable whose expected value we wish to go to zero as time approaches infinity. For definiteness, we choose $r_{d}(W)=$ $-c V_{d}$, where $c>0$ is a suitable real number, and $\mathcal{W}_{d}=$ $\{(-,, 0)\}$, which consists only of the trivial exosystem, so that $(P \wedge C) \wedge W=(P \wedge C) \boxplus(-,, 0)=P \wedge C$.

Theorem 4.1: (Stabilization/Regulation): If there exists a controller $C \in \mathcal{C}$ and non-negative observable $V_{d} \in \mathcal{A}_{P} \otimes \mathcal{A}_{c}$ such that the plant-controller network $P \wedge C$ satisfies

$$
\mathcal{G}_{P \wedge C}\left(V_{d}\right)+c V_{d} \leq 0
$$

for some real $c>0$, then $\left\langle V_{d}(t)\right\rangle \rightarrow 0$ as $t \rightarrow \infty$ exponentially for any plant-controller state.

This theorem follows from the stability results give in Section III-B.

Our next result is a theorem concerning nonlinear quantum $H^{\infty}$ robust control, which generalizes the linear quantum results given in [20], [23]. We use an exosystem class $\mathcal{W}_{d}=\{W$ : $W=(I, w, 0): w$ commutes with $\left.\mathcal{A}_{P}\right\}$ to describe the "disturbance" inputs. The next theorem is a consequence of a slight extension of the Bounded Real Lemma (Theorem 3.7) applied to the plant-controller network $P \wedge C$.

Theorem 4.2: ( $H^{\infty}$ Control): If there exists a controller $C \in$ $\mathcal{C}$ and a non-negative observable $V_{d} \in \mathcal{A}_{P} \otimes \mathcal{A}_{c}$ such that the plant-controller network $P \wedge C$ satisfies

$$
g^{2}-Z^{\dagger} Z \geq 0
$$




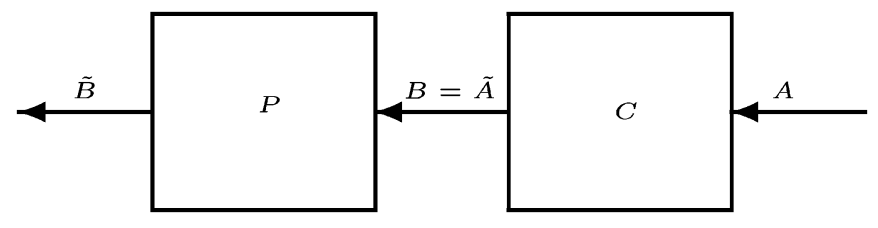

Fig. 7. Plant-controller network $P \triangleleft C$ for Examples 4.3, 4.4.

and

$$
\mathcal{G}_{(P \wedge C) \wedge W}\left(V_{d}\right)-g^{2} w^{\dagger} w+(N+Z w)^{\dagger}(N+Z w)-\lambda \leq 0
$$

for some real $g>0, \lambda \geq 0$, and all exosystem parameters $W \in \mathcal{W}_{d}$, then the plant-controller network $P \wedge C$ has gain $g$.

\section{Design Examples}

In this section we provide some simple examples to illustrate several issues concerning feedback control design of quantum systems. The examples (Examples 4.3, 4.4) employ the series architecture shown in Fig. 7.

Our first design example is a regulation problem analogous to the classical problem of designing a controller to maintain a given value of capacitor charge in a RC circuit, [30].

Example 4.3: (Regulation): Consider an optical cavity $P=$ $(1, a, 0)$ (a damped open harmonic oscillator, Example 3.10). If the input field is a vacuum, photons initially in the cavity will eventually leak out. Suppose our control objective is to maintain a given non-zero value for the steady state expected photon number. Let's choose a value $\alpha$ for the desired steady-state value of $a$, which corresponds to a number $\alpha^{*} \alpha$ of photons (i.e., we want the cavity to be in a coherent state $|\alpha\rangle$ in the steady-state, Appendix B).

Perhaps the simplest thing to do is to provide a source of fresh photons that can be supplied to the cavity to replace those that are lost. This might be achieved using a laser source or modulator $C=(1, \nu, 0)$ connected in series, as in Fig. 7. Here, $\nu$ is a complex number describing the strength of the source, and is to be determined, if possible.

We set $V_{d}=(a-\alpha)^{*}(a-\alpha)=a^{*} a-\alpha^{*} a-a^{*} \alpha+\alpha^{*} \alpha$, and for a positive real number $c, r_{d}(W)=-c V_{d}$, with $\mathcal{W}_{d}=$ $\{(-,, 0)\}$, which consists only of the trivial exosystem, as in Theorem 4.1. Note that the expected value of $V_{d}$ in the state $\alpha$ is zero: $\left\langle\alpha\left|V_{d}\right| \alpha\right\rangle=0$.

The design problem is to select $\nu$, a complex number, such that

$$
\mathcal{G}_{P \triangleleft C}\left(V_{d}\right)+c V_{d} \leq 0
$$

for suitable $c>0$. Then from (19) the LHS of this expression is $-a^{*} a(1-c)+a\left(\left(\alpha^{*} / 2\right)-\nu^{*}-c \alpha^{*}\right)+a^{*}((\alpha / 2)-\nu-$ $c \alpha)+\alpha^{*} \nu+\alpha \nu^{*}+c \alpha^{*} \alpha$. If we set $c=1 / 2, \nu=-\alpha / 2$, then this expression equals $-V_{d} / 2$. Therefore $\mathcal{G}_{P \triangleleft C}\left(V_{d}\right) \leq-V_{d} / 2$, which implies that the expected value of $V_{d}(t)$ tends to zero as $t \rightarrow \infty$ (by Theorem 3.5), and the control objective is achieved (notes also that the expected value of $P \triangleleft C$ in the cavity coherent state $|\alpha\rangle$ is zero: $\left\langle\alpha\left|\mathcal{G}_{P \triangleleft C}\left(V_{d}\right)\right| \alpha\right\rangle=0$ ).

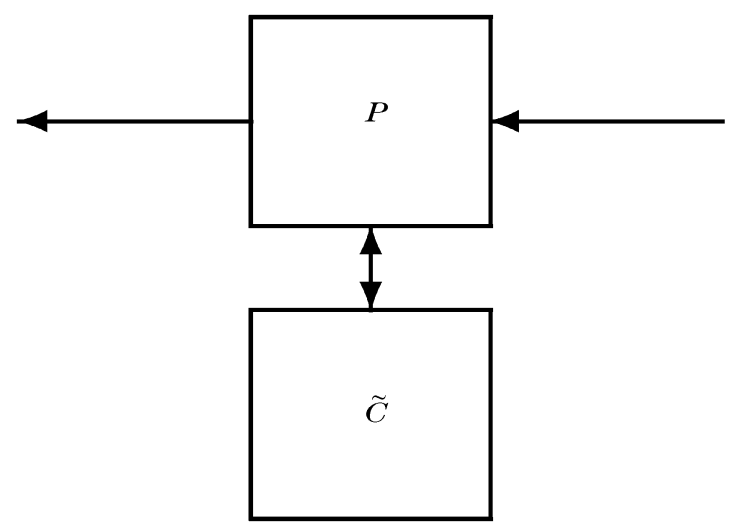

Fig. 8. Alternate representation of the plant-controller network $P \triangleleft C$ in the form $P \boxplus \tilde{C}$ for Example 4.3.

The effect of the controller is to place the cavity input field in a coherent state $|\nu\rangle$. It is well known (e.g. [13]) that this is equivalent to adding a Hamiltonian term to the cavity model and setting the input to vacuum. In the notation of this paper, this follows from (18) and (19): $\mathcal{G}_{P \triangleleft C}(X)=\mathcal{G}_{P \boxplus \tilde{C}}(X)$, where $\tilde{C}=\left({ }_{-},, i\left(\nu^{*} a-\nu a^{*}\right)\right)$. This is illustrated in Fig. 8 .

The next example shows that care must be exercised when attempting to use classical control design methods. Furthermore, one must take account of quantum noise and the fact that physical quantities do not in general commute.

Example 4.4: (Stabilization): Suppose we wish to stabilize the marginally stable system $P=\left(1, a+a^{*}, 0\right)$, a special case of the open oscillators of Example 3.10, using an approach analogous to a standard method from classical control theory for stabilizing Hamiltonian systems, [35, sec. 4.1].

Consider the series plant-controller network of Fig. 7. From Example 3.10, we know that this system is passive; if $C=$ $(1, u, 0)$ then

$$
\mathcal{G}_{P \triangleleft C}\left(V_{0}\right)=\mathcal{L}_{u}\left(V_{0}\right)+u^{*} Z+Z^{*} u+1
$$

where $Z=a^{*}-a$ and $V_{0}=a^{*} a$ (recall (30), (52)) with $W=$ $C)$. The classical Hamiltonian stabilization procedure suggests that we set $u=-k Z$ for some non-negative gain $k$. In order to implement this feedback, the controller $C$ needs to have access to the variable $Z$. However, $Z$ is not available in the output signal $\tilde{B}$, since by the output relation (15) $d \tilde{B}=L d t+d B$, where $L=a+a^{*}$.

We suppose that the plant can be re-engineered to have a second field channel which contains $Z$. Specifically, we consider the augmented system $P \boxplus C$, where $C=(1,-k Z, 0)$. The desired variable is now available in the second output of the augmented system, and so we can form the series connection $P \triangleleft C=\left(1,(1+k) a+(1-k) a^{*}, i k\left(\left(a^{*}\right)^{2}-a^{2}\right)\right)$, Fig. 9 . However, an examination of the dynamics of the quadratures $q=a+a *$ and $p=-i\left(a-a^{*}\right)$ shows that the feedback system $P \triangleleft C$ is marginally stable and not asymptotically stable for all $k \geq 0$ (the feedback system has poles at 0 and $-4 k$ ). Physically, neither of the field couplings $L=a+a^{*}=q$ and $-k Z=k\left(a-a^{*}\right)=i k p$ are sufficient for strict passivity and hence asymptotic stability. 


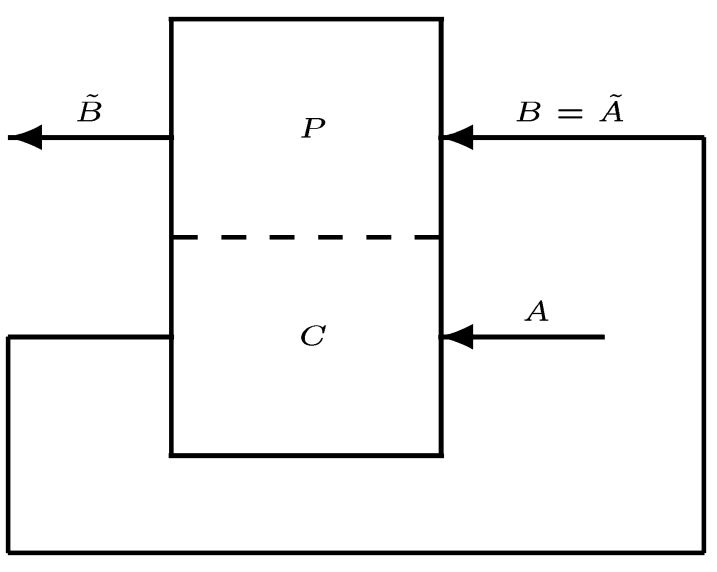

Fig. 9. Alternative representation of the plant-controller network $P \triangleleft C$ for Example 4.4.

A preferable stabilization scheme would be to replace $C$ by $\tilde{C}=(1, k a, 0)$. Then the re-engineered systems $P \boxplus C$ and $P \triangleleft \tilde{C}$ are both strictly passive and asymptotically stable. $h$ fill $\square$

\section{CONCLUSION}

In this paper we have extended J.C. Willems' theory of dissipative systems to open quantum systems described by quantum noise models. With the aid of recently developed methods for describing quantum feedback networks, we have shown how to describe external influences as arising from interactions with exosystems. The fundamental dissipation property was expressed in these terms. We presented an infinitesimal characterization of the dissipation property, which generalizes the well-known Positive Real and Bounded Real Lemmas. We also showed how to implement Willems' "control by interconnection" for open quantum systems using quantum network representations. We believe that the results in this paper provide useful methods for the analysis and design of quantum dissipative systems, and indeed networks of such systems. The quantum network based results we have presented are quite powerful, and merit further development. The network paradigm is particularly important if quantum technology is to move from the device and small system level to a more complex system level such as is being contemplated, for example, in the quantum computing community.

\section{APPENDIX}

\section{A. Two Level Atom (Qubit)}

The simplest quantum system has two energy levels and is often used to model ground and excited states of atoms. Since the advent of quantum computing, this system is also known as the qubit, the unit of quantum information. The two level atom is illustrated in Fig. 10(a), showing the action of the raising $\sigma_{+}$ and lowering $\sigma_{-}$operators. The Hilbert space for this system is $\mathrm{H}=\mathbf{C}^{2}$, the two-dimensional complex vector space. The

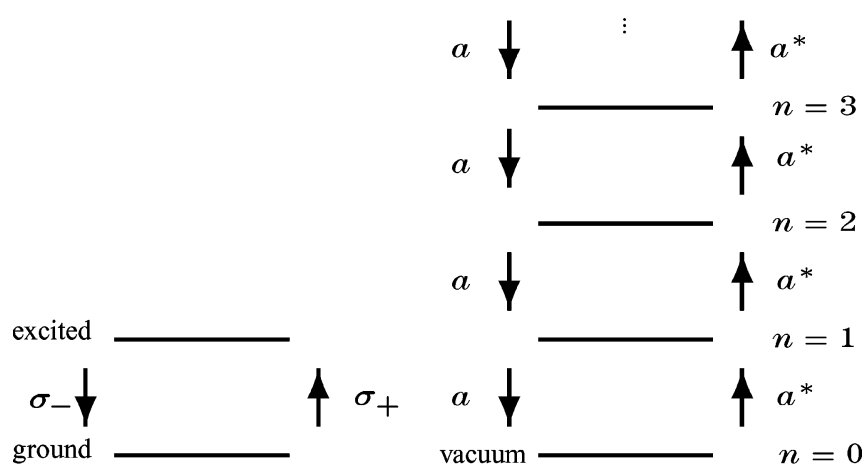

(a)

(b)

Fig. 10. Energy level diagrams. (a) Two-level atom (qbit). (b) Harmonic oscillator.

physical variable space $\mathcal{A}$ for this system is spanned by the Pauli matrices [26, sec. 2.1.3], [13, sec. 9.1.1]:

$$
\begin{aligned}
& \sigma_{0}=I=\left(\begin{array}{ll}
1 & 0 \\
0 & 1
\end{array}\right), \quad \sigma_{x}=I=\left(\begin{array}{ll}
0 & 1 \\
1 & 0
\end{array}\right), \\
& \sigma_{y}=I=\left(\begin{array}{cc}
0 & -i \\
i & 0
\end{array}\right), \quad \sigma_{z}=I=\left(\begin{array}{cc}
1 & 0 \\
0 & -1
\end{array}\right) .
\end{aligned}
$$

The raising and lowering operators are defined by $\sigma_{ \pm}=$ $1 / 2\left(\sigma_{x} \pm i \sigma_{y}\right)$. The basic commutation relations are $\left[\sigma_{x}, \sigma_{y}\right]=2 i \sigma_{z},\left[\sigma_{y}, \sigma_{z}\right]=2 i \sigma_{x}$, and $\left[\sigma_{z}, \sigma_{x}\right]=2 i \sigma_{y}$. The energy levels correspond to the eigenvalues of $\sigma_{z}$.

\section{B. Quantum Harmonic Oscillator}

The quantum harmonic oscillator is one of the most important examples because of its tractability and application to modeling, [26, Box 7.2], [24, sec. 10.6], [13, sec. 4.1]. Models for the optical cavity and boson fields are based on the quantum harmonic oscillator. The quantum harmonic oscillator is illustrated in Fig. 10(b), which shows infinite ladder of energy levels and the action of the creation $a^{*}$ and annihilation $a$ operators. The Hilbert space for the quantum harmonic oscillator is $\mathrm{H}=L^{2}(\mathbf{R}, \mathbf{C})$, the vector space of square integrable functions defined on the real line. The physical variable space $\mathcal{A}$ for this system is defined in terms of the annihilation operator $a$, with $a^{*}$ the adjoint of $a$, and the canonical commutation relations $\left[a, a^{*}\right]=$ 1 . The action of the annihilation operator may be expressed as $(a \psi)(x)=x \psi(x)-i(d \psi / d x)(x)$ on a domain of functions (vectors) $\psi$ in $\mathrm{H}$. The eigenvalues of $a^{*} a$ are the numbers $0,1,2, \ldots$ (number of quanta), with corresponding eigenvectors denoted $\psi_{n}(n=0,1,2, \ldots)$ called $\{$ lem number states $\}$. We have $a \psi_{n}=\sqrt{n} \psi_{n-1}$ and $a^{*} \psi_{n}=\sqrt{n+1} \psi_{n+1}$. For a complex number $\alpha$, a coherent state is defined by $|\alpha\rangle=$ $\exp \left(-(1 / 2)|\alpha|^{2}\right) \sum_{n=0}^{\infty}\left(\alpha^{n} / \sqrt{n !}\right) \psi_{n}$ (Dirac notation), and satisfies the eigenvalue relation $a|\alpha\rangle=\alpha|\alpha\rangle$.

\section{Operator Orderings}

In this appendix we review some definitions and results concerning operator ordering. 
Let $A$ and $B$ be self-adjoint operators on a Hilbert space $\mathrm{H}$. Then by definition $A \geq 0$ means $\langle\psi, A \psi\rangle \geq 0$ for all vectors $\psi \in \mathrm{H}$. Using this, we say $A \geq B$ to mean $A-B \geq 0$.

Now fix $A$ and $B$ self-adjoint, and $C$ is arbitrary. Assume $w^{*} A w \leq B+w^{*} C+C^{*} w$ for all operators $w$ acting on $\mathrm{H}$. Then we claim that $A \leq 0$.

To verify this claim, suppose by contradiction there exists $\psi_{0} \in \mathrm{H}$ such that $\left\langle\psi_{0}, A \psi_{0}\right\rangle>0$.

Now set $w=\alpha I$, where $\alpha$ is an arbitrary real number. Now the hypothesis implies $\alpha^{2}\left\langle\psi_{0}, A \psi_{0}\right\rangle \leq\left\langle\psi_{0}, B \psi_{0}\right\rangle+\alpha\left\langle\psi_{0},(C+\right.$ $\left.\left.C^{*}\right) \psi_{0}\right\rangle$. Since $\alpha$ is arbitrary, this gives a contradiction, establishing the claim.

Now fix arbitrary operators $C$ and $D$. We can use a similar argument to show that if $w^{*} C+C^{*} w \leq D$ for all operators $w$ acting on $\mathrm{H}$, then $C=0$.

\section{ACKNOWLEDGMENT}

The authors wish to thank G. Zhang for his helpful comments.

\section{REFERENCES}

[1] B. Anderson and S. Vongpanitlerd, Network Analysis and Synthesis. Englewood Cliffs, NJ: Prentice-Hall, 1973.

[2] M. Armen, K. Au, J. Stockton, A. Doherty, and H. Mabuchi, "Adaptive homodyne measurement of optical phase," Phys. Rev. A, vol. 89, no. 13, p. $133602,2002$.

[3] G. Auletta, M. Fortunato, and G. Parisi, Quantum Mech.. Cambridge, U.K.: Cambridge Univ. Press, 2009.

[4] V. Belavkin, "On the theory of controlling observable quantum systems," Autom. Remote Control, vol. 44, no. 2, pp. 178-188, 1983.

[5] L. Bouten, R. Van Handel, and M. R. James, "An introduction to quantum filtering," SIAM J. Control Optim., vol. 46, no. 6, pp. 2199-2241, 2007.

[6] H. Carmichael, An Open Systems Approach to Quantum Optics. Berlin, Germany: Springer, 1993.

[7] H. J. Carmichael, "Quantum trajectory theory for cascaded open systems," Phys. Rev. Lett., vol. 70, no. 15, pp. 2273-2276, 1993.

[8] E. Davies, Quantum Theory of Open Systems. New York: Academic Press, 1976.

[9] C. D'Helon and M. R. James, "Stability, gain, and robustness in quantum feedback networks," Phys. Rev. A., vol. 73, p. 053803, 2006.

[10] A. C. Doherty and K. Jacobs, "Feedback-control of quantum systems using continuous state-estimation," Phys. Rev. A, vol. 60, p. 2700, 1999.

[11] C. W. Gardiner, "Driving a quantum system with the output field from another driven quantum system," Phys. Rev. Lett., vol. 70, no. 15, pp. 2269-2272, 1993.

[12] C. W. Gardiner and M. J. Collett, "Input and output in damped quantum systems: Quantum stochastic differential equations and the master equation," Phys. Rev. A, vol. 31, no. 6, pp. 3761-3774, 1985.

[13] C. W. Gardiner and P. Zoller, Quantum Noise. Berlin, Germany: Springer, 2000

[14] J. Gough and M. R. James, "Quantum feedback networks: Hamiltonian formulation," Commun. Math. Phys., vol. 287, pp. 1109-1132, 2009.

[15] J. Gough and M. R. James, "The series product and its application to quantum feedforward and feedback networks," IEEE Trans. Autom. Control, vol. 54, no. 11, pp. 2530-2544, Nov. 2009.

[16] M. Green and D. J. N. Limebeer, Linear Robust Cotrol. Englewood Cliffs, NJ: Prentice-Hall, 1995.

[17] D. Hill and P. Moylan, "The stability of nonlinear dissipative systems," IEEE Trans. Autom. Control, vol. AC-21, no. 5, pp. 708-711, Oct. 1976.

[18] D. Hill and P. Moylan, "Dissipative dynamical systems: Basic inputoutput and state properties," J. Franklin Inst., vol. 309, pp. 327-357, 1980.

[19] R. L. Hudson and K. R. Parthasarathy, "Quantum Ito's formula and stochastic evolutions," Commun. Math. Phys., vol. 93, pp. 301-323, 1984.
[20] M. R. James, H. Nurdin, and I. R. Petersen, “ $H^{\infty}$ control of linear quantum systems," IEEE Trans. Autom. Control, vol. 53, no. 8, pp. 1787-1803, Jul. 2008.

[21] G. Lindblad, "On the generators of quantum dynamical semigroups," Commun. Math. Phys., vol. 48, pp. 119-130, 1976.

[22] S. Lloyd, "Coherent quantum feedback," Phys. Rev. A, vol. 62, p. 022108, 2000.

[23] H. Mabuchi, "Coherent-Feedback Quantum Control With a Dynamic Compensator," in arXiv:0803.2007, 2008.

[24] E. Merzbacher, Quantum Mechanics, 3rd ed. New York: Wiley, 1998.

[25] P. J. Moylan, "Implications of passivity in a class of nonlinear systems," IEEE Trans. Autom. Control, vol. AC-19, no. 4, pp. 373-381, 1974.

[26] M. A. Nielsen and I. L. Chuang, Quantum Computation and Quantum Information. Cambridge, U.K.: Cambridge Univ. Press, 2000.

[27] H. Nurdin, M. R. James, and A. C. Doherty, "Network synthesis of linear dynamical quantum stochastic systems," SIAM J. Control Optim., vol. 48, no. 4, pp. 2686-2718, 2009.

[28] H. Nurdin, M. R. James, and I. R. Petersen, "Coherent quantum LQG control," Automatica, vol. 45, pp. 1837-1846, 2009.

[29] R. Ortega and M. W. Spong, "Adaptive motion control of rigid robots: A tutorial," Automatica, vol. 25, no. 6, pp. 877-888, 1989.

[30] R. Ortega, A. J. van der Schaft, I. Mareels, and B. Maschke, "Putting energy back in control," IEEE Control Syst. Mag., vol. 21, pp. 18-32, Apr. 2001.

[31] R. Ortega, A. J. van der Schaft, B. Maschke, and G. Escobar, "Interconnection and damping assignment passivity-based control of port-controlled Hamiltonian systems," Automatica, vol. 38, pp. 585-596, 2002.

[32] K. R. Parthasarathy, An Introduction to Quantum Stochastic Calculus. Berlin, Germany: Birkhauser, 1992.

[33] L. K. Thomsen and H. M. Wiseman, "Atom-laser coherence and its control via feedback," Phys. Rev. A, vol. 65, p. 063607, 2002.

[34] H. L. Trentelman and J. C. Willems, " $H_{\infty}$ control in a behavioral context: The full information case," IEEE Trans. Autom. Control, vol. 44, no. 3, pp. 521-536, Mar. 1999.

[35] A. J. van der Schaft, $L_{2}$-Gain and Passivity Techniques in Nonlinear Control. New York: Springer Verlag, 1996.

[36] R. van Handel, J. Stockton, and H. Mabuchi, "Feedback control of quantum state reduction," IEEE Trans. Autom. Control, vol. 50, no. 6, pp. 768-780, Jun. 2005.

[37] J. C. Willems, "Dissipative dynamical systems-Part I: General theory," Archive Rat. Mech. Anal., vol. 45, pp. 321-351, 1972.

[38] J. C. Willems, "On interconnections, control, and feedback," IEEE Trans. Autom. Control, vol. 42, no. 3, pp. 326-339, Mar. 1997.

[39] J. C. Willems, "Open dynamical systems and their control," Documenta Mathematica, vol. ICM(III), pp. 697-706, 1998

[40] J. C. Willems, "Control as interconnection," in Feedback Control, Nonlinear Systems and Complexity. New York: Springer, 2003.

[41] J. C. Willems and H. L. Trentelman, "Synthesis of dissipative systems using quadratic differential forms: Part I," IEEE Trans. Autom. Control, vol. 47, no. 1, pp. 53-69, Jan. 2002.

[42] H. Wiseman, "Quantum theory of continuous feedback," Phys. Rev. A, vol. 49, no. 3, pp. 2133-2150, 1994.

[43] H. M. Wiseman and G. J. Milburn, "All-optical versus electro-optical quantum-limited feedback," Phys. Rev. A, vol. 49, no. 5, pp. 4110-4125, 1994.

[44] W. Wiseman and G. Milburn, Quantum Measurement and Control. Cambridge, U.K.: Cambridge Univ. Press, 2010.

[45] M. Yanagisawa and H. Kimura, "Transfer function approach to quantum control-part I: Dynamics of quantum feedback systems," IEEE Trans. Autom. Control, vol. 48, no. 12, pp. 2107-2120, Dec. 2003.

[46] M. Yanagisawa and H. Kimura, "Transfer function approach to quantum control-part II: Control concepts and applications," IEEE Trans. Autom. Control, vol. 48, no. 12, pp. 2121-2132, Dec. 2003.

[47] B. Yurke and J. S. Denker, "Quantum network theory," Phys. Rev. A, vol. 29, no. 3, pp. 1419-1437, 1984.

[48] G. Zames, "On the input-output stability of time-varying nonlinear feedback systems Part I: Conditions derived using concepts of loop gain, conicity, and positivity," IEEE Trans. Autom. Control, vol. AC-11, no. 2, pp. 228-238, 1966.

[49] K. Zhou, J. Doyle, and K. Glover, Robust and Optimal Control. Englewood Cliffs, NJ: Prentice Hall, 1996. 


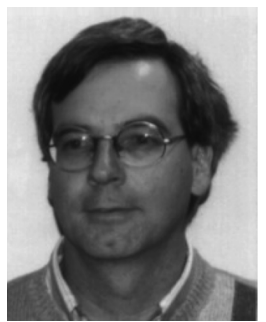

Matthew R. James (S'86-M'86-SM'00-F'02) was born in Sydney, Australia, in 1960. He received the B.Sc. degree in mathematics and the B.E. (with honors) in electrical engineering from the University of New South Wales, Sydney, Australia, in 1981 and 1983, respectively, and the Ph.D. degree in applied mathematics from the University of Maryland, College Park, in 1988.

In 1988/1989, he was a Visiting Assistant Professor with the Division of Applied Mathematics, Brown University, Providence, RI, and from 1989 to 1991 he was Assistant Professor with the Department of Mathematics, University of Kentucky, Lexington. In 1991, he joined the Australian National University, Australia, where he served as Head of the Department of Engineering during 2001 and 2002. He has held visiting positions with the University of California, San Diego, CA, Imperial College, London, U.K., and University of Cambridge, Cambridge, U.K. He previously worked on the SIAM Journal on Control and Optimization, Automatica, and Mathematics of Control, Signals, and Systems. His research interests include quantum, nonlinear, and stochastic control systems.

Dr James received the SIAM Journal on Control and Optimization Best Paper Prize for 2007. He is currently serving as Associate Editor for the IEEE TRANSACTIONS ON AUTOMATIC CONTROL. He held an Australian Research Council Professorial Fellowship during 2004 to 2008.

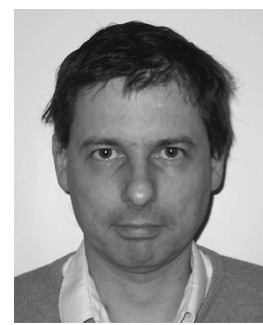

John E. Gough was born in Drogheda, Ireland, in 1967. He received the B.Sc. and M.Sc. degrees in mathematical sciences and the Ph.D. degree in mathematical physics from the National University of Ireland, Dublin, in 1987, 1988, and 1992, respectively.

From 1995 to 1996, he was a Visiting Researcher at the Volterra Centre, Rome, Italy, and from 1996 to 1999 was a Research Fellow at the National University of Ireland, Maynooth. He was a Reader in Mathematical Physics with the Department of Mathematics and Computing, Nottingham-Trent University, U.K., up until 2007 when he joined the Institute of Mathematics and Physics, University of Wales, Aberystwyth, as established Chair of Mathematics. His research interests include quantum probability and measurement and control of open quantum dynamical systems. 